\title{
Réflechir, rechercher, innover en chirurgie maxillo-faciale. Entretien avec Albino Triaca
}

\author{
Albino TRIACA ${ }^{1 *}$, Sophie ROZENCWEIG ${ }^{2}$ \\ 1 Klinik Pyramide am See, Bellerivestrasse 34, 8034 Zürich, Suisse \\ 21 rue Thiers, 38000 Grenoble, France
}

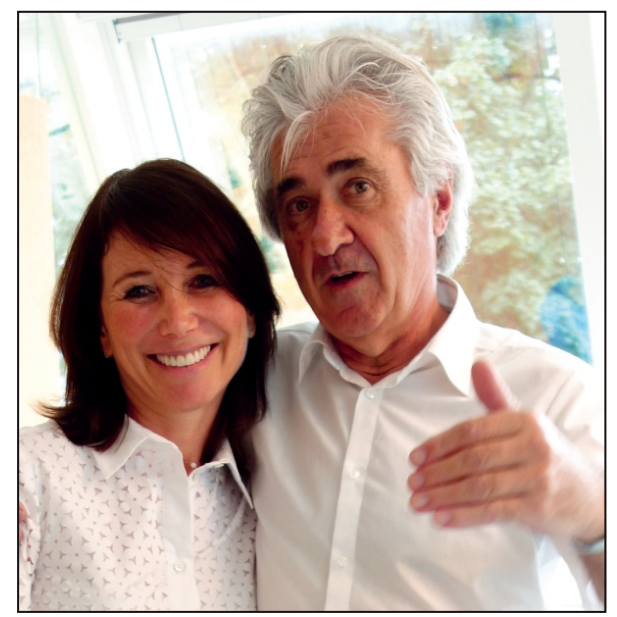

Albino Triaca a été chef de clinique au centre de chirurgie maxillo-faciale et d'esthétique de San Marin. Il est également depuis un an professeur du service de chirurgie maxillo-faciale de Salzbourg, où il dirige plus spécifiquement le service de la chirurgie du nez. II exerce depuis plus de 30 ans à la Klinik Pyramide au bord du lac de Zurich où il me reçoit pour cet entretien.
Albino Triaca me fait entrer dans son bureau et me dit d'emblée: "Je suis Albino, tu es Sophie, on se tutoie».

Le regard pétille, les idées fusent; mais à chaque question, il prend le temps de la réflexion et de l'écoute. Albino Triaca est volubile, inquiet de ne pouvoir transmettre tout son savoir " Tu as des inventions plein la tête, 10000 détails tous plus importants les uns que les autres, tu meurs et tout est perdu». Conférencier international, il a un peu diminué ses déplacements. "Jusqu'il y a quelque temps, je présentais des conférences dans 27 pays chaque année; maintenant, je me cantonne à 12 ». Sur les étagères s'étalent des crânes, mandibules, maxillaires, bassin, avec des prototypes d'interventions ou d'appareils. Les os sont parfois segmentés en toutes petites pièces assemblées par des minis-plaques avec autant de précision que celle d'un ébéniste. Et encore me dit-il, il s'agit d'anciens prototypes; tous les plus récents sont conservés à son domicile. Lorsque je l'interroge sur le fait qu'il n'ait que peu publié par rapport à la quantité de

* Auteur pour correspondance : triaca@smile.ch techniques qu'il a développées, il me répond avec un soupçon de frustration: "Je n'ai pas le temps... Parfois, je me rends compte, et cela m'attriste que mes idées et techniques sont reprises par d'autres sans même que je ne sois cité. Maintenant que je travaille pour le service de Salzbourg, les étudiants vont publier mes travaux».

Entre les questions, Albino me fait le plaisir d'assister à ses consultations pré- ou post-opératoires. Cela nous permet d'échanger sur l'analyse des demandes du patient, sur l'examen de son visage, de sa fonction, sur les approches thérapeutiques, les difficultés, les résultats. Pour Albino, il y a des solutions à tous les problèmes : "Les idées me viennent la nuit. La nuit, la plupart des individus dorment. C'est à ce moment-là que mon cerveau est le plus efficace et que je trouve des solutions... C'est peut-être parce que je mange beaucoup de chocolat..."

Les auditeurs d'Albino Triaca ont été éblouis par ses présentations lors des réunions scientifiques de la SFODF à Toulouse et à Aix-en-Provence. Pour les lecteurs de l'Orthodontie Française, nous espérons rendre hommage à cet homme passionné en vous faisant mieux connaître ses travaux et vous faire 
partager son esprit de génie, en attendant de venir l'écouter lors de la conférence de prestige qu'il va présenter pour la SFODF, en mai $2017^{1}$.

Sophie Rozencweig : Albino, nous avons eu la chance de t'entendre lors des réunions scientifiques de la SFODF à Toulouse en 2014 et à Aix-en-Provence en 2015. Tu nous as présenté quelques unes de tes innovations en matière de chirurgie maxillo-faciale (CMF) mais tu n'as pas eu le temps de développer tous les sujets que tu aurais aimé aborder. Nous souhaiterions que cet entretien soit pour les lecteurs de l'Orthodontie Française l'occasion de connaître ta vision d'une part sur ce que la CMF peut apporter aujourd'hui (et demain) à nos patients en termes d'équilibre esthétique et fonctionnel, d'autre part sur la façon dont tu envisages la collaboration entre l'orthodontiste et le chirurgien maxillo-facial.

$\mathrm{Tu}$ es un guide et un pionnier pour de très nombreux chirurgiens maxillo-faciaux. As-tu eu, toimême, un mentor? Peux-tu nous raconter ton parcours professionnel?

Albino Triaca : Quand j'étais jeune, j'étais passionné par les soudures. J'achetais des carcasses de voiture et je m'amusais à souder les différentes pièces jusqu'à réparer les voitures complètement. Mon rêve était de devenir designer. À 18 ans, j’ai voulu m'inscrire à l'Institut de Design de Florence, mais je ne me suis pas senti accepté, peut-être parce que j'étais suisse et qu'alors il existait des tensions entre la Suisse et l'Italie. Alors, j'ai fait médecine à Zurich. $\mathrm{Au}$ fur et à mesure de mes études, il m'est apparu comme une évidence que je voulais m'occuper de la face. Alors, j'ai fait dentaire. J'ai travaillé chez Obwegeser. Puis chez Millard à Miami avec qui j'ai appris la chirurgie plastique, et plus spécifiquement celle du nez. Obwegeser et Millard ont été mes deux mentors. J'ai toujours eu plein d'idées. Rares sont les personnes, surtout lorsque ce sont d'éminentes personnes, qui te laissent exposer et développer tes idées. Obwegeser et Millard m’ont non seulement laissé exprimer mes concepts mais ils m'y ont même encouragé ; ils étaient fiers de moi. Ils ont été plus que des mentors pour moi, ils ont été de vrais pères. Ils m'ont montré l'exemple et m'ont donné confiance.

S.R. : Tu as adapté des techniques existantes, tu en as créé d'autres. Est-ce la recherche de l'harmonie qui te conduit à innover?

${ }^{1}$ Plus de renseignements sur le site ouvert à partir d'octobre 2016 : http://sfodf-prestige2017.com

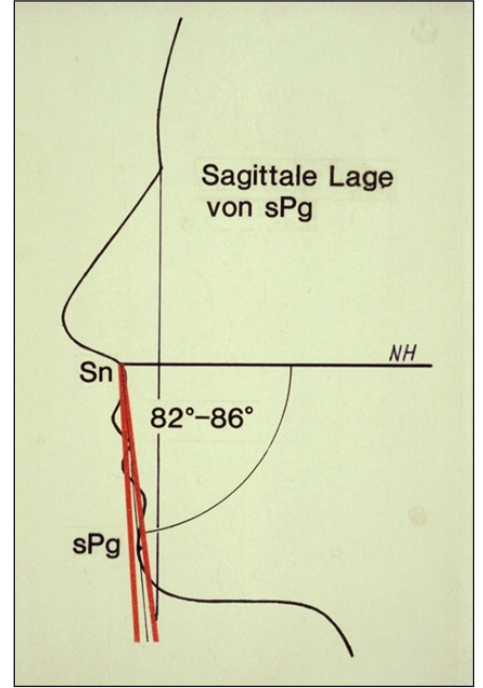

Figure 1

La ligne faciale est pour Triaca une des références de l'équilibre du profil. Elle est de 82 à $86^{\circ}$ par rapport au plan de Camper, lui-même perpendiculaire au plan central d'Andresen.

A.T. : Oui, la recherche de l'harmonie à la fois esthétique et fonctionnelle, et la recherche de solutions aux problèmes que je ne parviens pas à résoudre avec les techniques existantes. Chacun a sa sensibilité. Pour mon oeil à moi, l'harmonie faciale tient parfois à de petits détails : le support de la lèvre, la visibilité du bord incisif, une encoche mentonnière marquée, un bord mandibulaire bien dessiné, etc.

SR: Lorsque tu effectues une nouvelle intervention, es-tu inquiet? En informes-tu ton patient?

AT : Bien sûr, il y a toujours une appréhension à réaliser un nouveau geste sur un être humain. Lorsque j'imagine une nouvelle intervention, je choisis un patient que je connais très bien et je le préviens.

S.R. : Tu as décrit la face comme étant constituée de segments : nez, lèvre supérieure, lèvre inférieure, menton. Quels sont tes outils diagnostiques pour étudier les rapports, les proportions, l'harmonie de la face? Utilises-tu des repères céphalométriques?

A.T. : Chaque segment doit être harmonieux non seulement pris isolément mais aussi en considérant l'ensemble des segments. Mon guide céphalométrique est la «ligne faciale». Elle définit la position sagittale des segments. Elle présente une angulation de 82 à $86^{\circ}$ par rapport à la ligne horizontale de Moorees [7]. Si le nez est important, l'angulation est plus proche des $86^{\circ}$. Si la face est convexe, l'angulation est de $82^{\circ}$ (Fig. 1). 


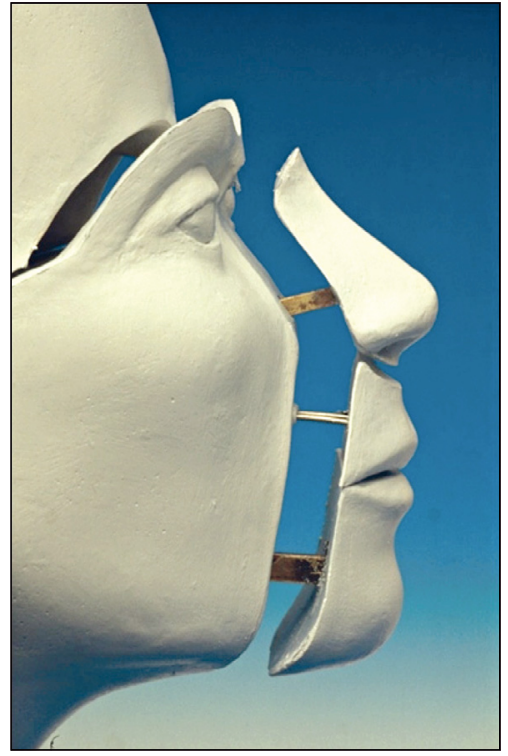

Figure 2

Modèle des annexes de Haab.

Mon principal outil diagnostique est inspiré du modèle créé par le designer Haab qui a travaillé 10 ans dans le service d'Obwegeser. Il réalisait des moulages en plâtre des annexes : nez, lèvre supérieure, lèvre inférieure, menton qu'il déplaçait pour figurer le résultat final (Fig. 2), ce que je fais aujourd'hui avec Photoshop. J'ai modifié le modèle puisqu'avec la chirurgie du chin wing, je peux à présent travailler sur le bord basilaire mandibulaire (Fig. 3), mais ce modèle m'accompagnera toute ma vie, il est mon guide.

Quant à la dimension verticale, les critères les plus importants sont l'exposition des incisives centrales maxillaires et la compétence labiale.

S.R. : Comment intègres-tu les attentes et les désirs de tes patients dans tes plans de traitement?

A.T. : Le modèle des segments de la face est très parlant pour expliquer au patient les objectifs que je me suis fixés en fonction de mes préférences, de mon regard. Je propose et le patient dispose. Si le patient désire telle ou telle variation du montage Photoshop (Fig. 4) que je lui présente, on échange, je modifie mes objectifs. Aujourd'hui, en Europe, les personnes aiment les faces très carrées. Régulièrement, des patientes au profil extrêmement convexe me consultent. De plus en plus souvent, ces patientes désirent une avancée mandibulaire bien plus importante que celle que je leur aurai proposée. Ces patientes auront un profil presque concave après

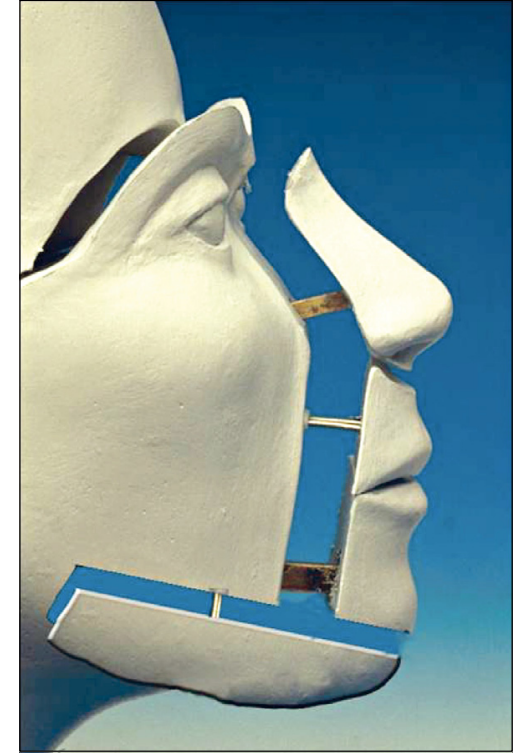

Figure 3

Modèle de Haab modifié par Triaca (l'intervention de chin wing permettant de modeler le bord basilaire de la mandibule).

l'intervention (Fig. 5), mais c'est leur désir et je le respecte. En Asie, par contre, les femmes préfèrent les visages le plus fin possible.

S.R. : Albino me présente une patiente qui vient de Russie, qui a bénéficié de deux traitements orthodontiques successifs et se plaint de douleurs au niveau de son articulation gauche.

A.T. : Tu vois cette patiente Elvira (Fig. 6) habite Moscou. Elle a bénéficié de deux traitements successifs de compensation de sa classe III squelettique. Elle vient aujourd'hui parce qu'elle présente des douleurs articulaires à gauche mais également parce qu'elle n'aime pas l'angulation de sa lèvre supérieure qui marque ses sillons nasogéniens. On voit bien, à l'examen cone beam (Fig. 7), les différences morphologiques entre les deux condyles. Avancer le maxillaire permettra de redonner de l'espace au niveau articulaire. La compensation de la classe III squelettique, en forçant le recul mandibulaire provoque la version compensatoire des incisives maxillaires et l'éversion de la lèvre supérieure. C'est le résultat des précédents traitements. Mon plan de traitement, ce sera la mise en place d'une gouttière occlusale, la décompensation orthodontique, puis je réévaluerai. Je suis sûr que redonner de l'espace au condyle pour supprimer la compression au niveau intra-capsulaire va lui redonner une liberté de mouvement et une fonction sans douleur. 


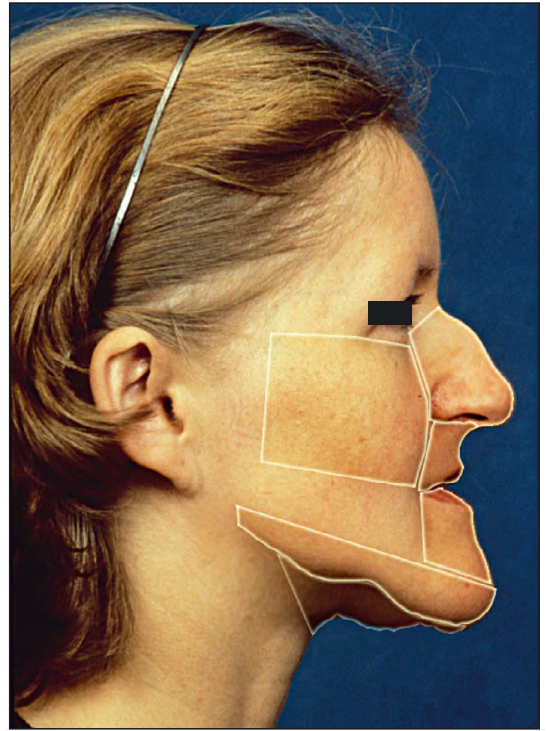

Figure 4

Modélisation de la face en différents segments. Chaque segment de la face peut-être déplacé grâce à Photoshop, permettant ainsi une interaction avec le patient pour comprendre ses attentes et permettre au chirurgien d'exprimer ses préférences.

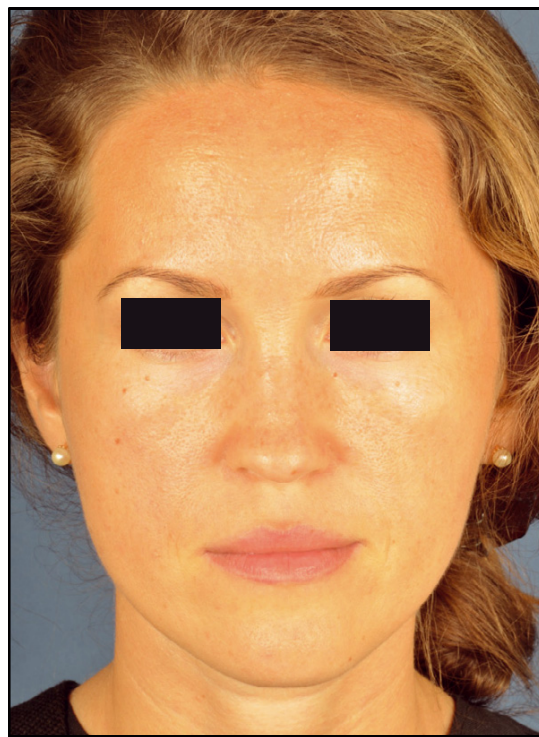

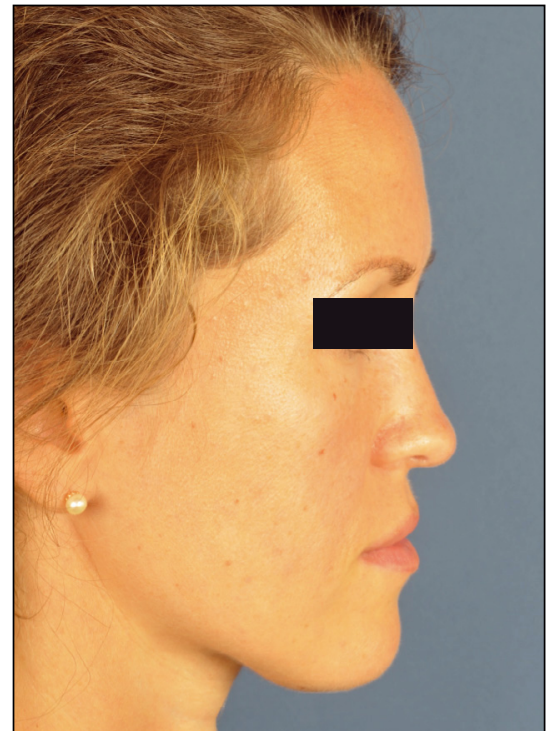

b

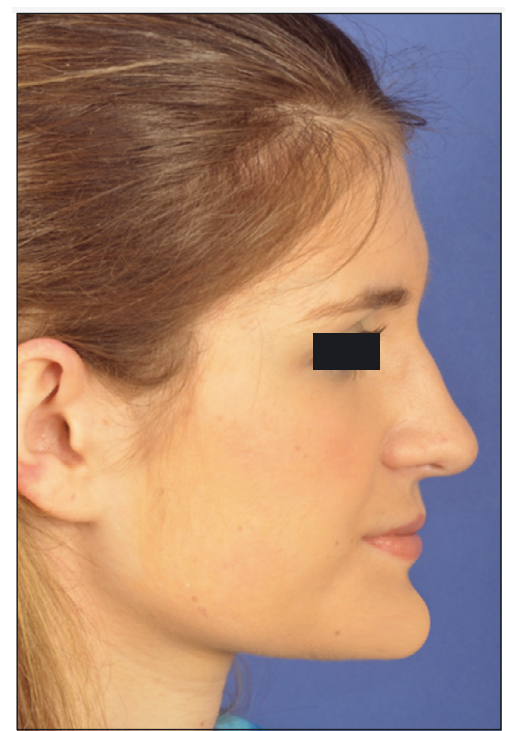

Figure 5

Exemple de déplacement du segment «bord basilaire » par Photoshop. Ici, avancée excessive simulée résultant en une concavité trop marquée du profil.

Figure 6

Photographies extra-buccales d'Elvira. (a) Vue de face, (b) vue de profil et (c) tête levée. Noter la déficience de l'étage moyen de la face et la déviation du menton vers la gauche.

S.R. : Lorsque je pose la question de ce qui me semble être une déficience de tout l'étage moyen, de l'asymétrie faciale, de l'éventualité d'une avancée par Le Fort II, Albino me répond:

A.T. : Mais ce n'est pas la demande de la patiente! S'il s'agissait d'une demande de la patiente, je ferais un Le Fort I et je modifierai les pommettes avec du bio os fixé à l'aide de membranes. Je n'ai ni recours à des implants qui risquent de créer des résorptions osseuses, ni au Le Fort II. Cette intervention est pour moi réservée aux grands syndromes. Si tu décales d'un dixième de millimètre le rebord orbitaire, tu 


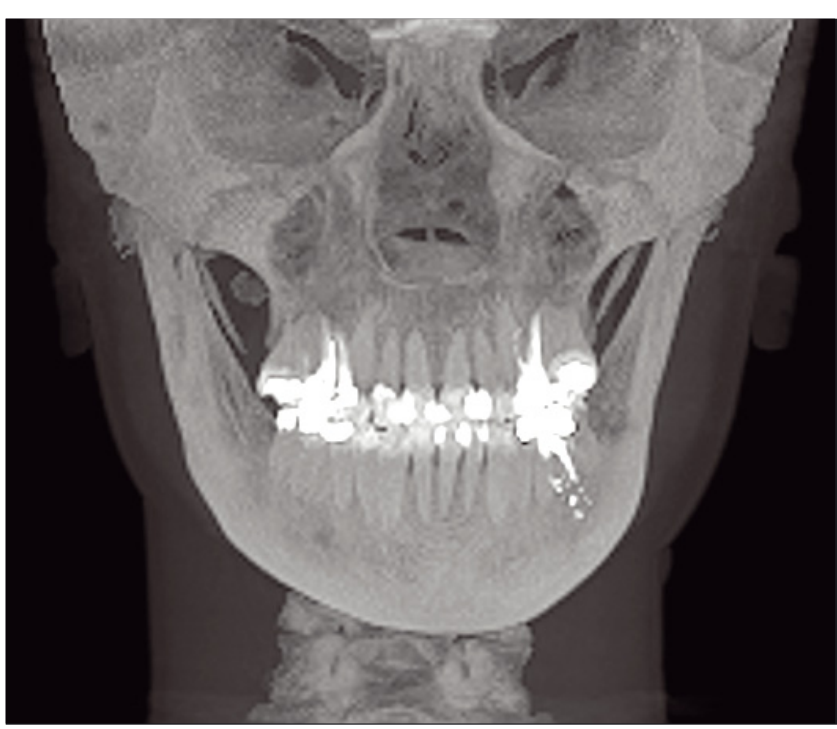

a

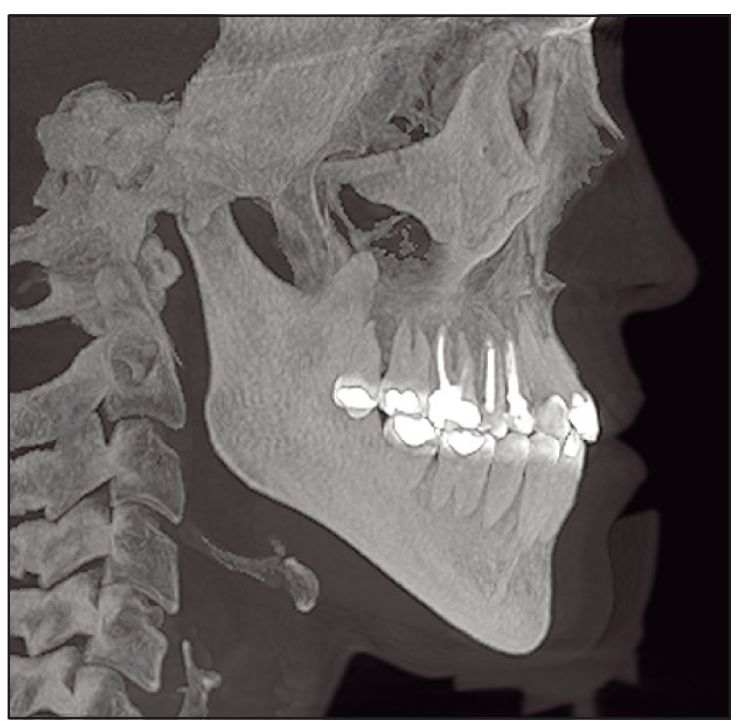

b

Figure 7

Cone beam d'Elvira. (a) Vue de face, (b) vue de profil.

affectes le fonctionnement de l'œil de façon irréversible et c'est une catastrophe.

S.R. : N'estimes-tu pas délicat de montrer tes objectifs thérapeutiques en photographie?

A.T. : Effectivement, certains praticiens objectent que c'est risqué pour le cas où tu n'atteindrais pas le résultat escompté avec exactitude. Mon point de vue est qu'il est très important que le chirurgien montre au patient quelle est sa propre préférence. En revanche, je fais signer au patient une décharge comme quoi il s'agit d'une proposition et non d'une garantie du résultat final. Lors de la chirurgie, si je me rends compte que je ne peux réaliser un avancement de $9 \mathrm{~mm}$ mais finalement de $6 \mathrm{~mm}$, la priorité est d'ordre médical et fonctionnel.

S.R. : Certains patients ont-ils le blues après l'intervention? Te créent-ils des problèmes?

A.T. : Pour moi, l'important est de rendre les gens satisfaits de leur image. Parfois, j'ai des patients qui dépriment après l'intervention. Sur près de 8000 interventions, il m'est effectivement arrivé d'avoir quelques patients insatisfaits même si le résultat était très proche de celui planifié. Avec l'âge, j'essaie d'avoir le nez pour les patients à ennuis, le patient qui te pollue ta journée, t'appelle sans cesse. Parfois, je préconise de consulter un psychiatre. Ce dernier m'aide soit à accompagner le ou la patiente, soit à décliner la proposition d'intervention. Un jour, j'ai reçu une jeune patiente, et j'ai senti qu'il ne fal- lait pas que je l'opère. Les parents, l'orthodontiste ont fait pression sur moi. J'ai alors fait signer un avenant comme quoi, en cas d'insatisfaction, je réinterviendrai et replacerai la mandibule là où elle était avant l'intervention. Finalement, tout s'est bien passé. Si le patient signe tous les documents de décharge et de consentement éclairé, je n'ai pas de problème avec la loi.

Pour la plupart des patients, même s'ils ont quelques difficultés à s'accepter juste après, dans les deux mois qui suivent, avec les réactions positives de l'entourage, ils sont heureux. Souvent, la personne la plus difficile à gérer sur le plan psychologique est la maman du patient. Je comprends cela. Le plus souvent, je parviens à la réconforter.

S.R. : Selon toi, ce qui est beau est souvent fonctionnel. L'augmentation de volume des voies aériennes, la qualité du sommeil sont pour toi des questions fondamentales. Régularité, symétrie, proportions. . . Les critères d'équilibre fonctionnel et esthétique sont-ils toujours compatibles? As-tu été confronté à des cas où les objectifs esthétiques et fonctionnels ne pouvaient être réunis en même temps?

A.T. : Oui, tout à fait. Je t'ai parlé des rapports et des proportions des différents segments de la face et de ma référence céphalométrique, la "facial line ». Ma troisième référence est la compétence labiale. La plupart des praticiens ne parlent que de proportions, de nombre d'or, de valeurs standards. Je ne me fie 


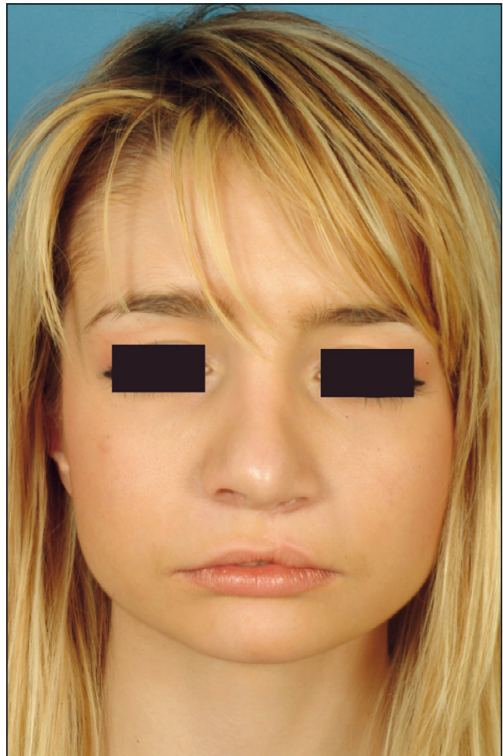

a

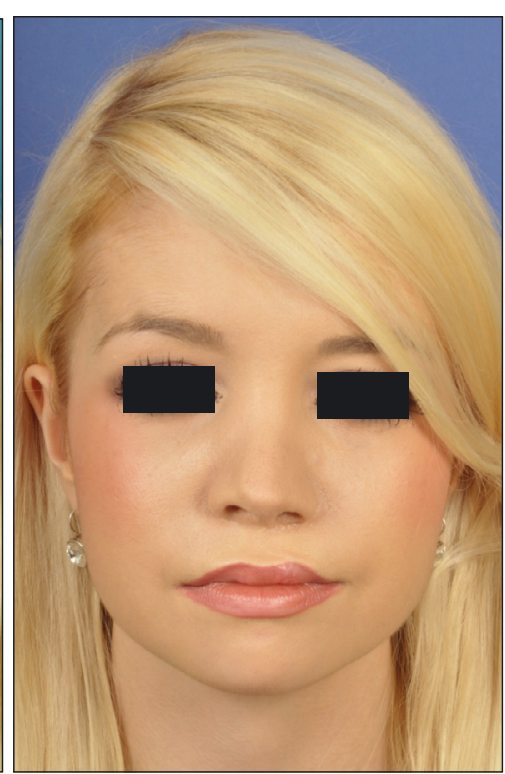

b

Figure 8

Photographie extra-buccale avant (a) et après intervention (b). L'augmentation de hauteur de l'étage inférieur du visage doit respecter l'équilibre fonctionnel. Le rapport idéal des lèvres, de 1 pour la lèvre supérieure pour 2 pour la lèvre inférieure, doit dans certains cas être abandonné au profit de la compétence labiale. Ici le rapport est de 1 pour 1,3 parce qu'au-delà on aurait provoqué une incompétence labiale.

pas à tout cela. Par exemple, si l'on parle du rapport idéal des lèvres, il est de 1 pour la lèvre supérieure pour 2 pour la lèvre inférieure. Chez certains patients, tu obtiendras un rapport de 1/1,3 parce qu'audelà tu crées une incompétence labiale. Ma priorité, c'est la compétence labiale (Fig. 8).

S.R. : Pour maintenir ce que tu appelles le "volume ", c'est-à-dire le soutien des téguments de la face, tu préfères envisager des traitements sans extractions. Pourtant, le maintien d'une occlusion dentaire stable amène parfois l'orthodontiste à prescrire des extractions. Comment envisages-tu le dialogue avec l'orthodontiste pour décider de ce choix?

A.T. : Mon choix est toujours de maintenir le volume dans le sens transversal et antéropostérieur, mais également de décomprimer l'espace intracapsulaire des articulations temporomandibulaires (ATM).

Selon moi, l'augmentation de volume va dans le sens de l'esthétique, la réduction de volume dans le sens de l'inesthétique et du risque d'altérations fonctionnelles (apnée du sommeil) (Fig. 9).

Parfois l'occlusion dentaire nous conduit à des extractions. Lorthodontiste me fait part de ses priorités et on en discute. Il faut alors informer le patient que le volume perdu devra éventuellement être compensé chirurgicalement par une ostéotomie de Le Fort I. A la mandibule, je préfère éviter les extractions dans les cas de classe II car cela augmente encore le surplomb incisif et les risques de résorption condylienne liés à une ostéotomie sagittale de trop grande amplitude. On peut parfois éviter les extractions en envisageant le traitement autrement (Fig. 10) grâce à la distraction antéropostérieure du segment frontal qu'elle soit maxillaire ou mandibulaire [4-6, 9] (Figs. 11a, 11b) mais également grâce à la distraction transversale maxillaire (Fig. 12) et mandibulaire (Fig. 15) [3].

S.R. : Résous-tu ainsi le manque de tissu de soutien en regard des incisives mandibulaires?

A.T. : Oui, bien sûr. Lorsqu'il existe un fort encombrement, l'orthodontiste peut hésiter à niveler l'arcade mandibulaire au risque de créer des déhiscences. Lorsque tu réalises une distraction alvéolaire du segment antérieur, un peu au-delà de ce qui est nécessaire, que l'orthodontiste mobilise les dents dans l'os néoformé, tu récrées une couverture d'os alvéolaire et de gencive attachée en regard des incisives (Figs. 13, 14)

S.R. : Combien de millimètres d'expansion transversale estimes-tu réalisables? 


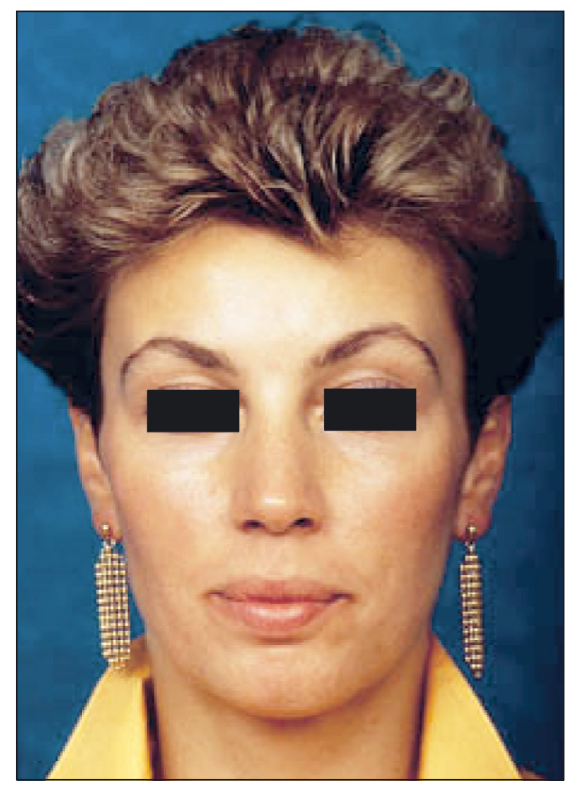

a

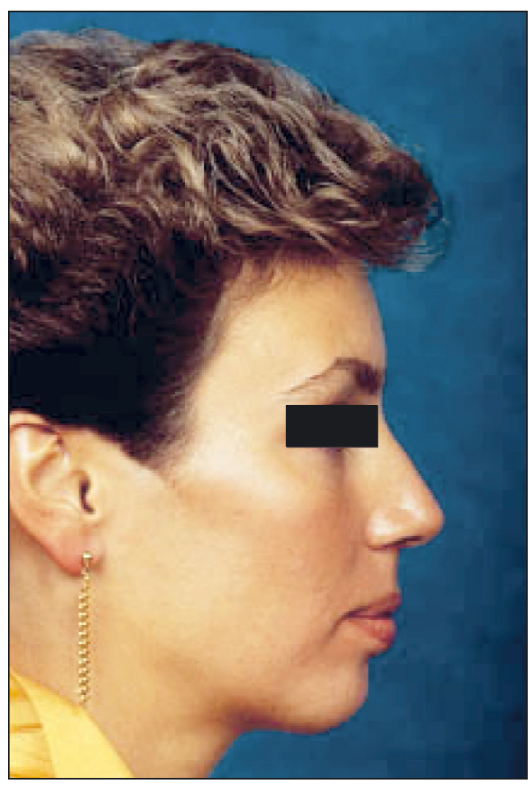

b

Figure 9

Le traitement d'un décalage de classe II par compensation dento-alvéolaire (réduction de volume) n'est pour Triaca ni esthétique, ni fonctionnel. (a) Vue de face, (b) vue de profil.

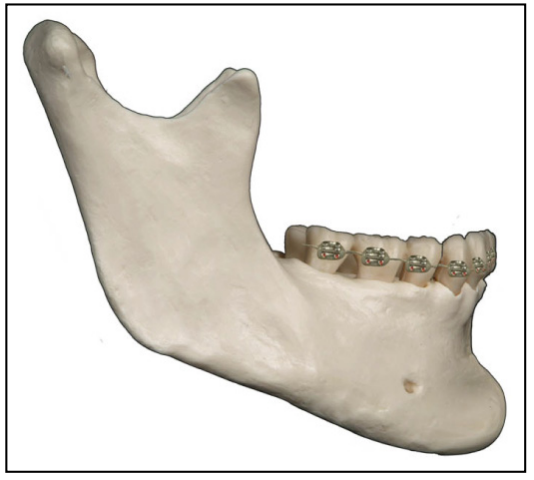

a

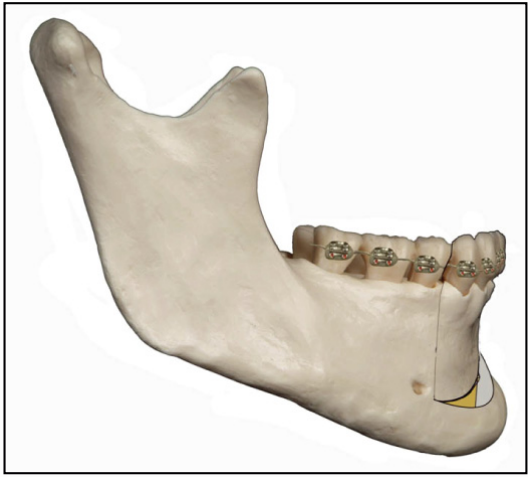

b

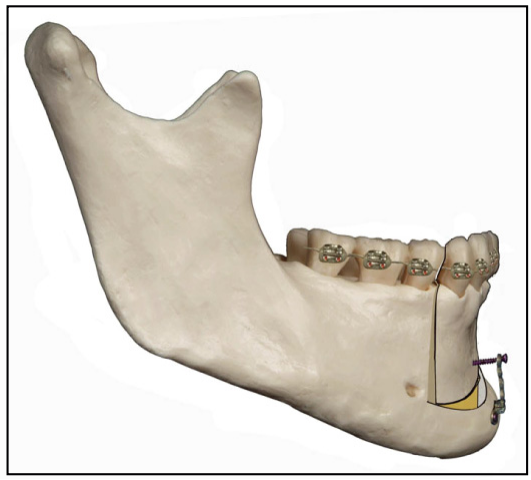

c

Figure 10

$(\mathrm{a}-\mathrm{c})$ Ostéotomie segmentaire du segment frontal mandibulaire.

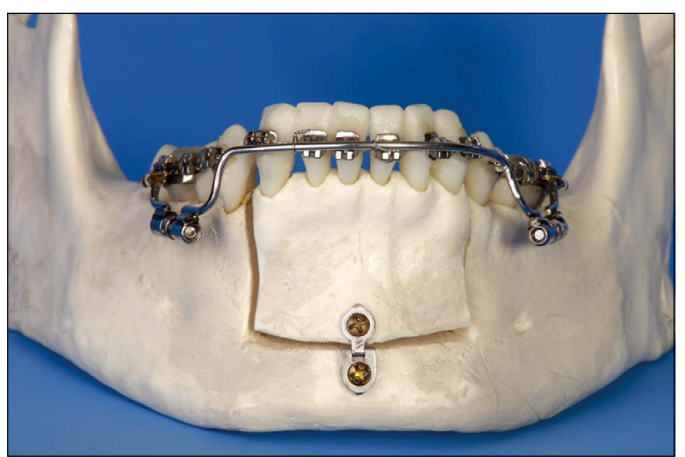

a

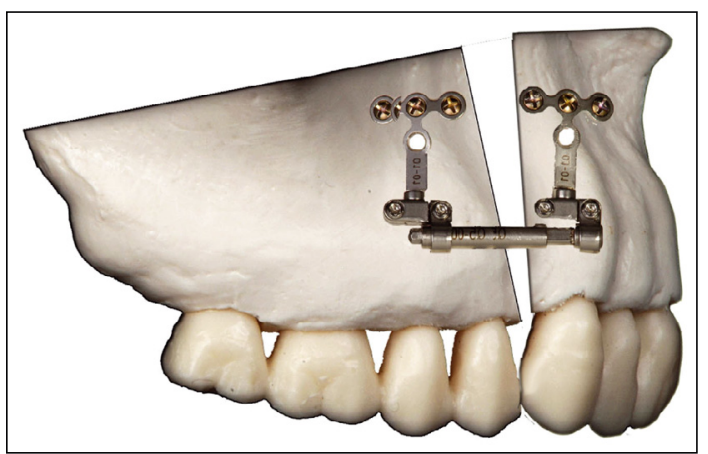

b

Figure 11

Distraction alvéolaire du segment frontal mandibulaire (a) et du segment frontal maxillaire (b). 


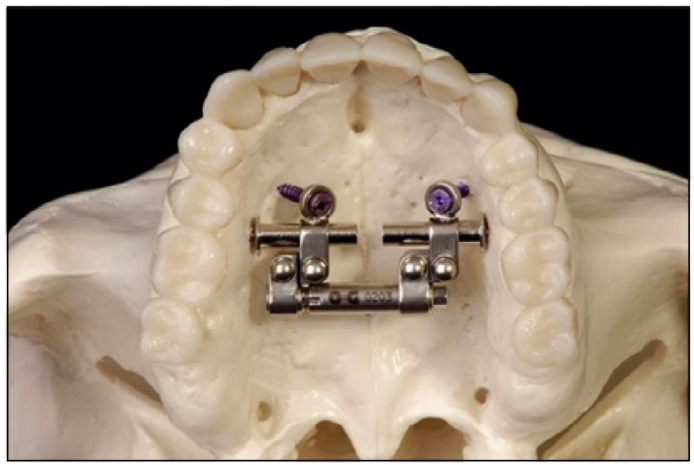

Figure 12

Distraction transversale maxillaire.

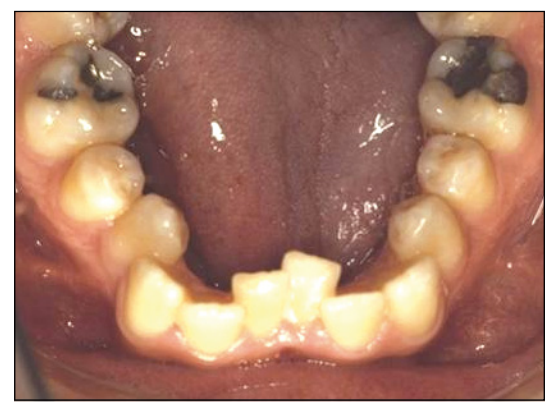

a

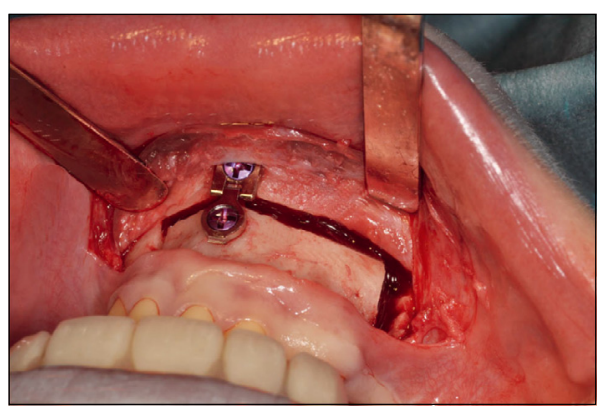

b

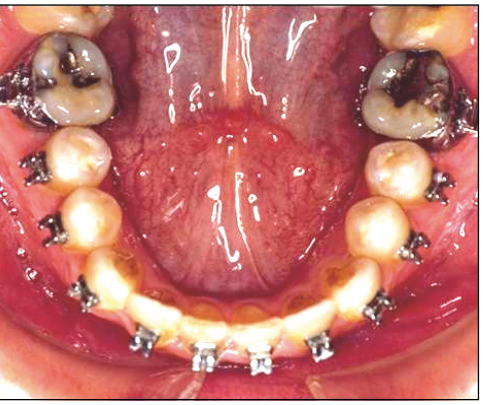

c

Figure 13

La distraction du segment frontal a permis de corriger l'encombrement sans avoir recours aux extractions. (a) Vue intra-buccale avant traitement, (b) distraction du segment frontal et (c) vue intra-buccale après traitement.

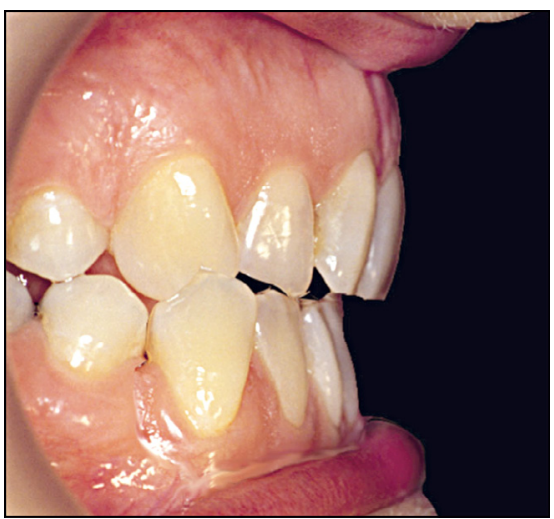

a

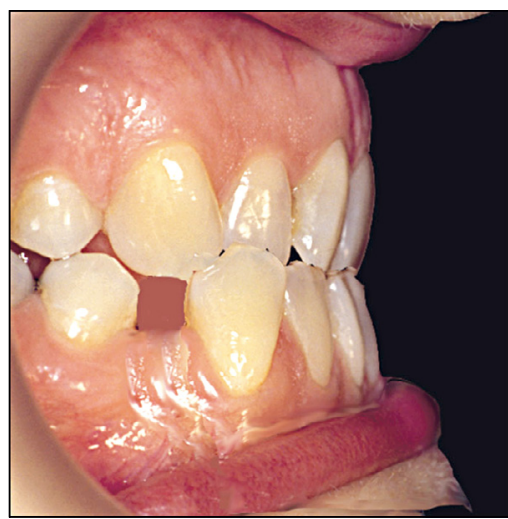

b

Figure 14

La distraction du segment frontal un peu au-delà de ce qui est nécessaire permet, de par la fermeture d'espace qui s'ensuit, de récréer un environnement osseux et gingival satisfaisant pour les incisives mandibulaires. (a) Vue intra-buccale avant distraction et (b) après.

A.T. : Gunbay T, et al. [3] ont montré qu'une expansion moyenne de $6,48 \mathrm{~mm}$ dans le sens transversal provoquait une rotation disto-latérale du condyle de 2,5 degrés à 3 degrés (calculée au CT scans) (Fig. 15e et 15f).
Guerrero a montré que l'on pouvait aller jusqu'à $10 \mathrm{~mm}$ d'expansion transversale [2]. Pour ma part, j'estime que l'on peut difficilement excéder 5-6 mm sans provoquer des douleurs articulaires. Encore une fois, cela dépend du patient. Il m'est arrivé d'envi- 


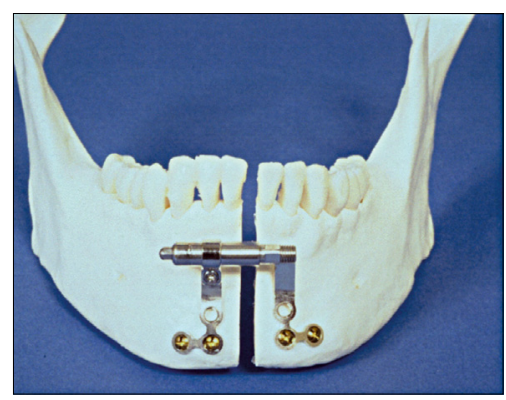

a

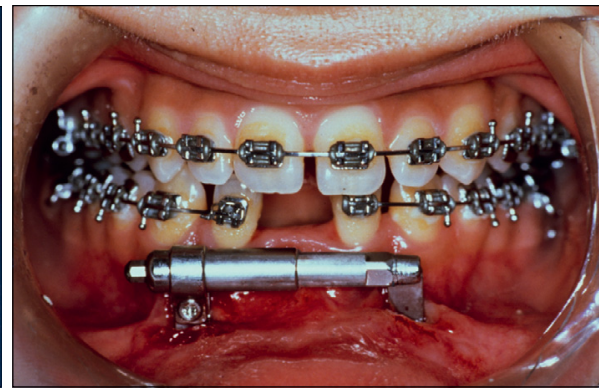

b

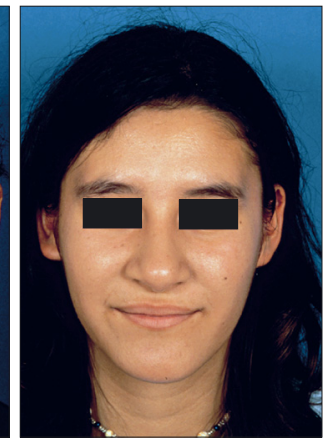

d

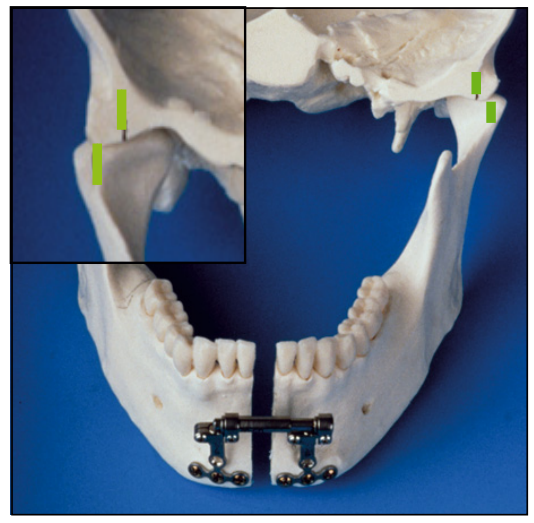

f

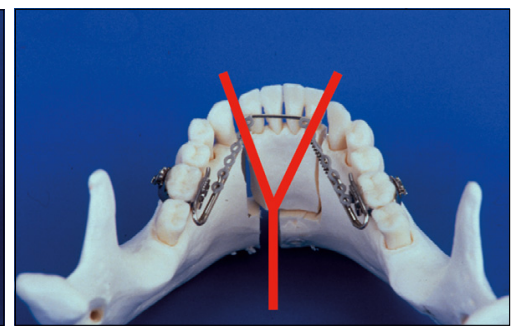

h

Figure 15

Distraction transversale mandibulaire. (a) Distracteur vissé sur le corpus mandibulaire, sur modèle. (b) Distracteur en photographie intra-buccale. L'espace des deux incisives mandibulaires manquantes a été réouvert. Photographies extra-buccales avant (c) et après (d) distraction. Noter l'arrondissement du contour mandibulaire. Impact articulaire de la distraction mandibulaire, avant (e) et après ( $f$ ) distraction. Les condyles se déplacent du même nombre de millimètres que la zone antérieure. ( $g$ et $h$ ) La distraction en $\mathrm{Y}$ permet d'augmenter transversalement la boîte à langue tout en corrigeant l'encombrement par la distraction du segment frontal. 
sager une distraction symphysaire de $8 \mathrm{~mm}$ sur un enfant de 11 ans qui a bien supporté.

Je privilégie la distraction du segment frontal pour corriger l'encombrement sauf s'il est nécessaire de travailler sur le sens frontal afin d'augmenter transversalement la boîte à langue. La tension générée par le distracteur peut être relâchée en le dévissant légèrement pour éviter une transposition latérale du condyle. En conclusion, j'effectue très rarement une distraction transversale seule; je l'accompagne toujours d'une distraction sagittale; il s'agit en fait d'une découpe mandibulaire en trois pièces (Figs. 15g, 15h).

En conclusion, il faut distinguer la distraction de Guerrero où toute la mandibule est élargie transversalement, ce qui a un impact sur les ATM de ma distraction qui est strictement alvéolaire (Fig. 15).

S.R. : Tu as de plus en plus souvent recours à la distraction mandibulaire. Peux-tu nous décrire cette intervention $[12,16]$ ? Combien de temps maintiens-tu les distracteurs en place?

A.T. : J'ai placé mon premier distracteur mandibulaire il y a 15 ans.

Chez le jeune, la distraction osseuse sagittale est plutôt bien supportée. Ce n'est pas forcément le cas chez l'adulte mais elle présente de nombreux avantages : avancements importants, reconstruction osseuse, absence de risque d'altération condylienne (Figs. 16 à 18). Lorsqu'un patient a présenté des phénomènes de résorptions condyliennes, mais que les condyles sont corticalisés, il est nettement préférable, selon moi, d'envisager une distraction plutôt qu'une ostéotomie sagittale. Les distracteurs sont adaptés au plus prêt du contour gingival et des dents. C'est le patient qui l'active. Le distracteur est activé de trois fois $1 / 4$ de $\mathrm{mm}$ matin, midi et soir. Une fois une légère sur-correction obtenue, le distracteur est maintenu trois mois en bouche.

S.R. : La difficulté de contrôler la position condylienne en cours d'intervention, le risque d'altérations idiopathiques temporomandibulaires représentent une limite et un risque de la CMF. Tu nous as dit lors de la réunion scientifique de Toulouse et d'Aix-enProvence que la prévention de ces atteintes consiste en une meilleure planification thérapeutique. Peux-tu développer cet argument?

A.T. : Lorsque j'exerçais dans le service d'Obwegeser, une journée entière par semaine était réservée

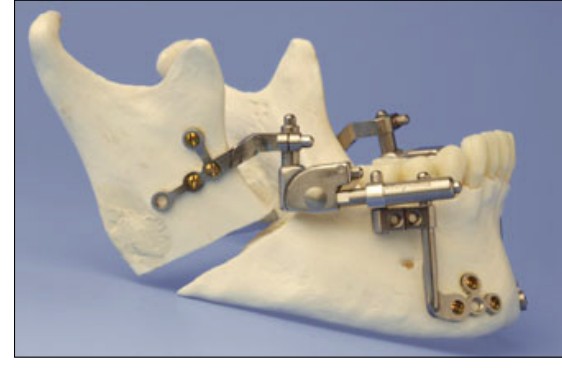

Figure 16

Distracteur mandibulaire.

uniquement pour les patients opérés et souffrants de douleurs articulaires.

Par le passé, j'ai réalisé des ostéotomies sagittales de plus de $10 \mathrm{~mm}$. Je me suis rendu compte qu'audelà de $8 \mathrm{~mm}$, on avait de forts risques d'altérer le condyle. Lorsque l'avancement est important, je préfère avoir recours à la distraction mandibulaire qui, comme je l'ai dit plus haut, n'altère pas le condyle et est bien plus stable dans le temps.

Par ailleurs, selon moi, ce n'est pas tant le contrôle de la position condylienne en cours d'intervention qui est important, mais l'absence de compression. Il est fondamental de ne pas comprimer le condyle.

S.R. : Pour contrôler la forme, la position du bord mandibulaire et du menton, la symétrie, la compétence labiale, tu as modifié la technique de Grimm décrite en 1961 [1] et proposé il y a six ans l'intervention de chin wing. Peux-tu nous décrire les spécificités de cette technique [13] ?

A.T. : Il s'agit d'une intervention simple avec peu de suites opératoires qui permet à la fois de recontourer l'angle et le bord basilaire mandibulaire dans tous les sens de l'espace (Figs. 19a-19h). Elle est également efficace pour aider à résoudre les problèmes d'apnées du sommeil. À la différence de la génioplastie décrite par Grimm, l'ostéotomie pour le wing est verticale. Il ne faut décoller le périoste qu'au niveau du trait d'ostéotomie. Tout le bord basilaire reste donc vascularisé par les attaches périostées. Cela est indispensable pour prévenir une résorption ultérieure. Contrairement au chin wing, les techniques de «chin degloving» conduisent à l'accumulation de parties molles sous-mentales; ceci génère une gêne à la fois esthétique, qui n'est pas tolérée par les patients et fonctionnelle, puisque l'on risque de créer une congestion des tissus para-pharyngés pouvant faire le lit d'apnées du sommeil. Sur le plan 


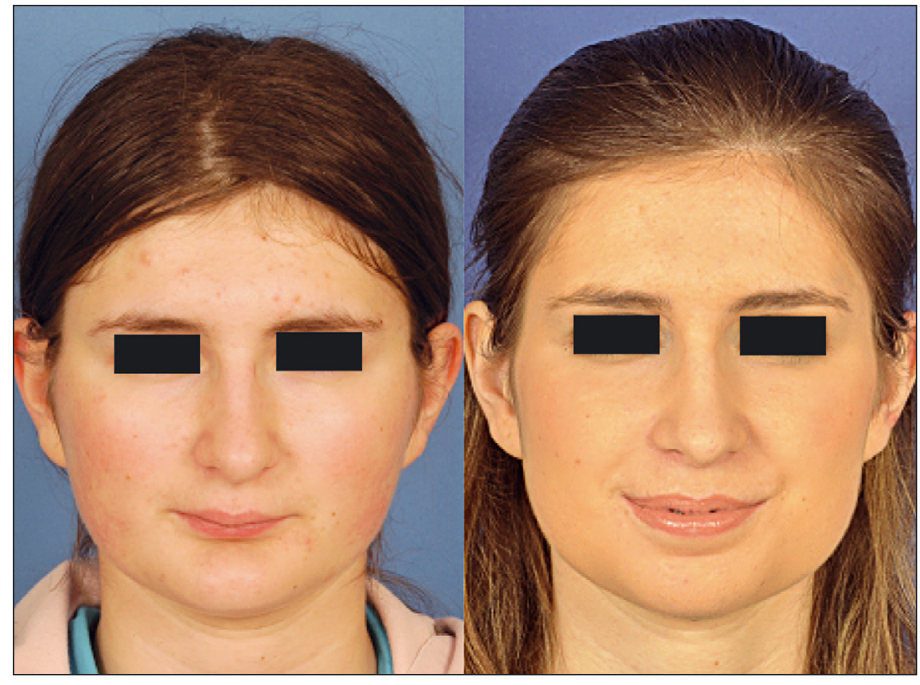

a

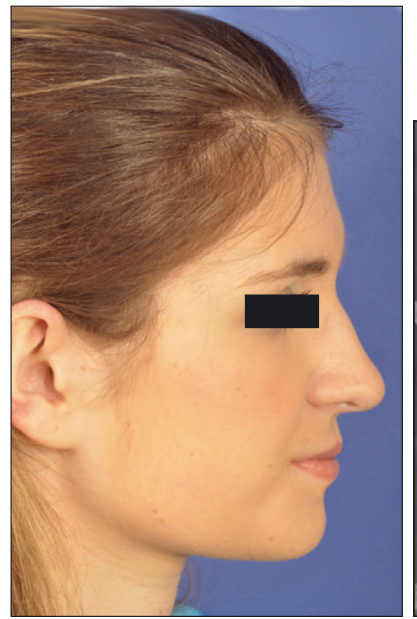

b

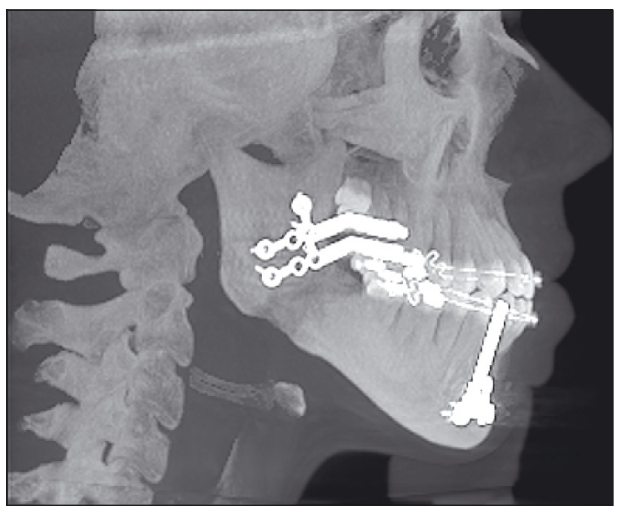

C

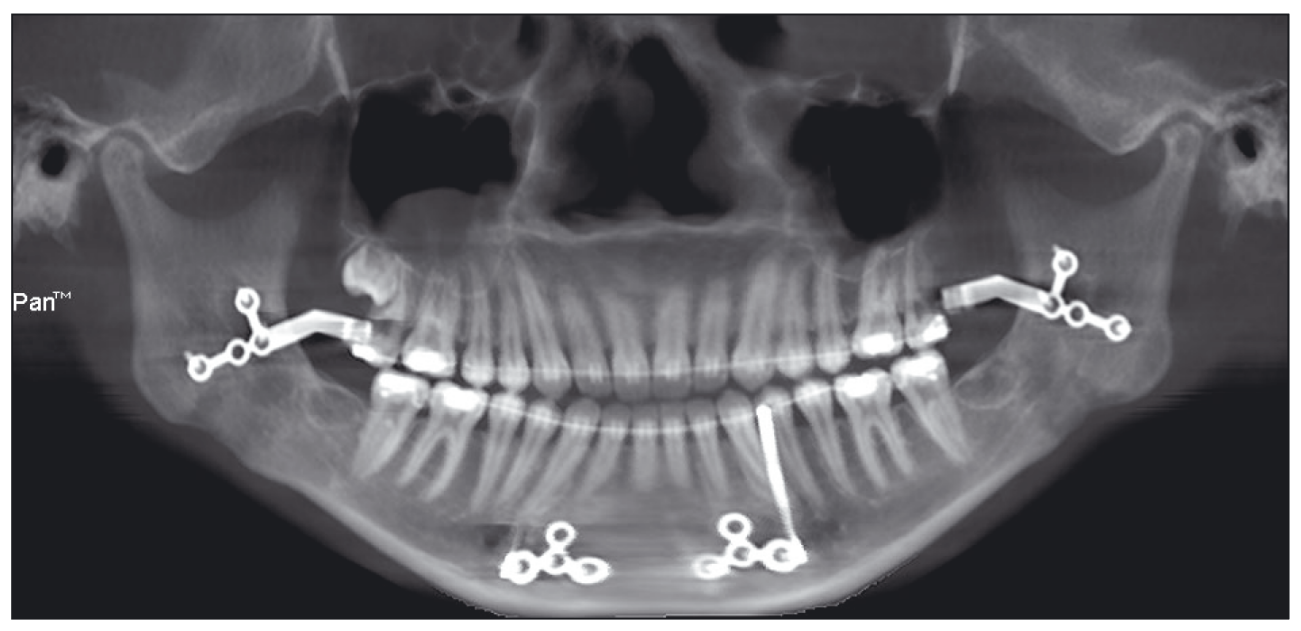

d

Figure 17

Cas $n^{\circ} 1$ d'avancement mandibulaire conséquent par distraction mandibulaire. (a) Vues extra-buccales avant et après distraction. Vue du profil (b), téléradiographie (c) et radiographie panoramique (d), après distraction. 


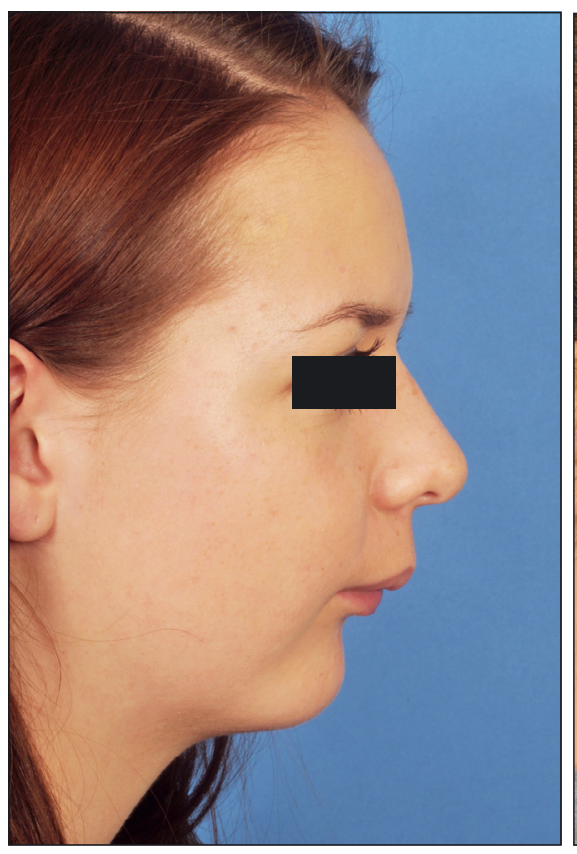

a

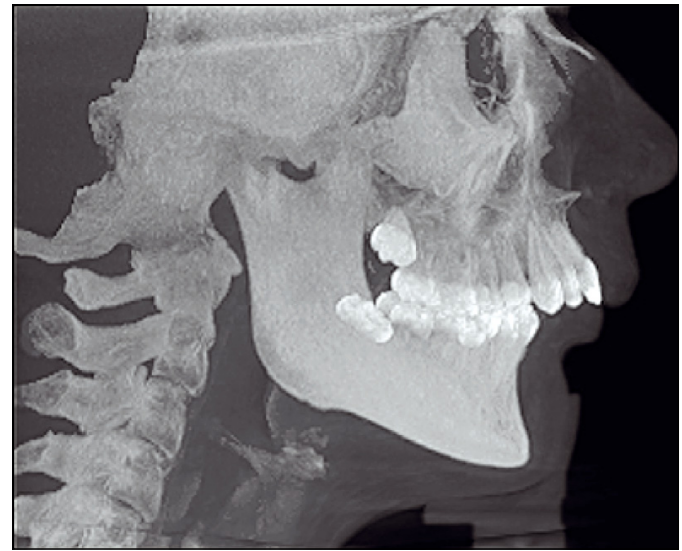

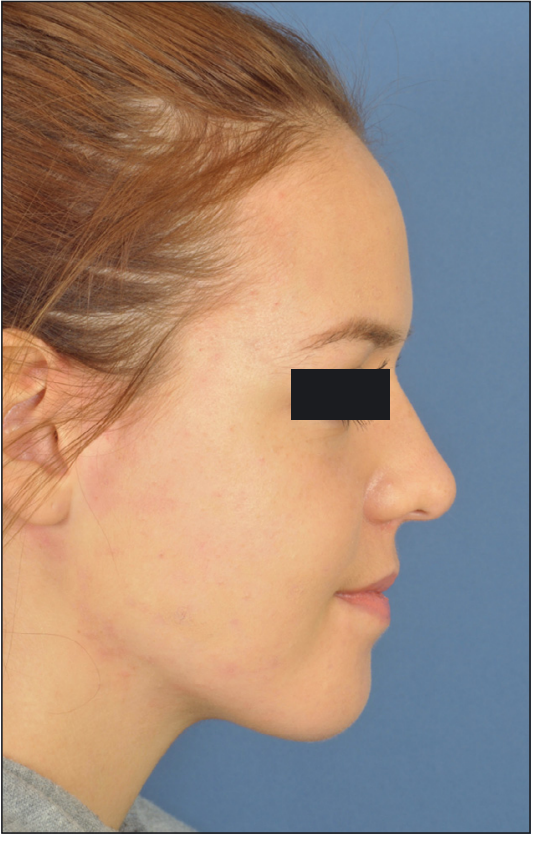

b

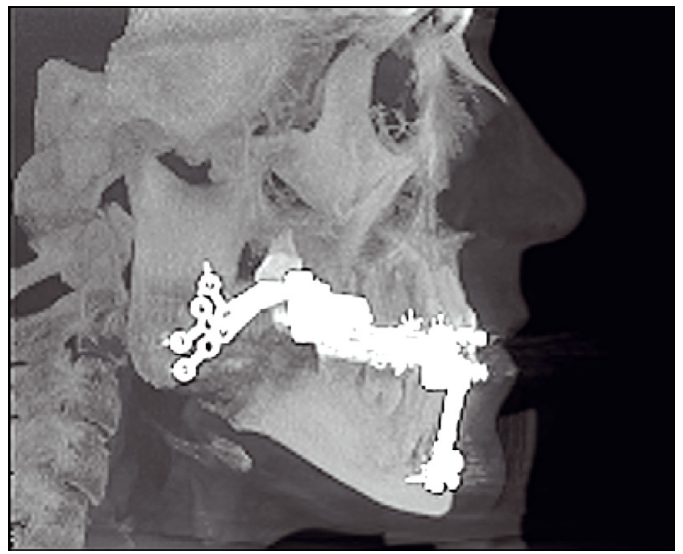

d

Figure 18

Cas $n^{\circ} 2$ d'avancement mandibulaire conséquent par distraction mandibulaire. Vues extra-buccales avant (a) et après (b) distraction. Téléradiographies de profil avant (c) et après (d) distraction.

fonctionnel, le chin wing permet également de rétablir une compétence labiale (Figs. 20, 21).

S.R. : Comment gères-tu le positionnement condylien en cours d'intervention?

A.T. : Je réalise un modèle en 3D qui préfigure l'avancement. Comme je réalise des avancées sans dérotation, il n'y a pas de risque de compression du condyle.

S.R. : Tu réalises donc des avancées mandibulaires par ostéotomie sagittale sans dérotation. Pourquoi? En revanche, peux-tu changer sans risque l'orientation $d u$ plan d'occlusion par la distraction alvéolaire du segment frontal dans les open-bite comme dans les deep-bite?
A.T. : Effectivement, je ne modifie pas l'orientation du plan d'occlusion mandibulaire avec l'ostéotomie sagittale, en tous cas, surtout pas dans le sens inverse des aiguilles d'une montre. La rotation antihoraire avec abaissement de l'angle crée un étirement de la sangle ptérygomassétérine et peut induire une compression des ATM (Fig. 22).

Si le patient présente une hyperdivergence squelettique, pour fermer la béance, j’aurai alors recours à une impaction maxillaire.

Une infraclusion antérieure simple peut être fermée par une distraction partielle verticale antérieure du segment frontal, surtout en cas d'inversion de la 

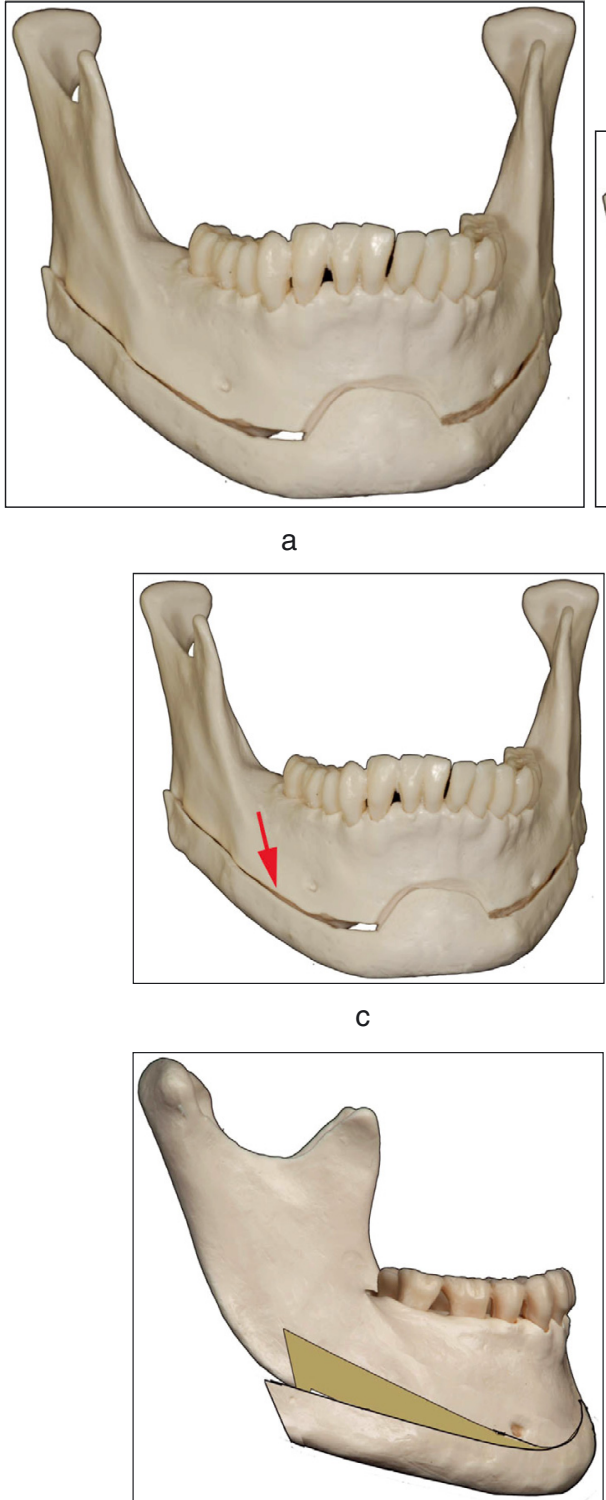

e

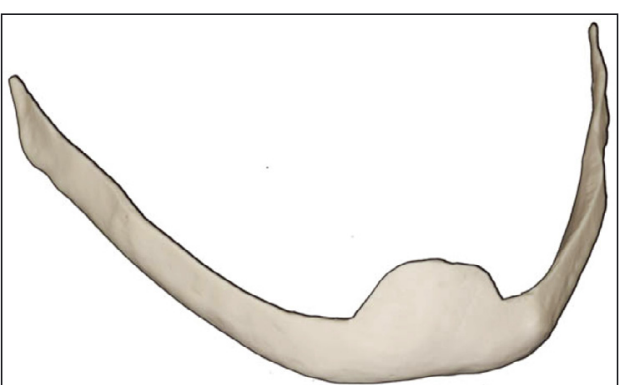

b

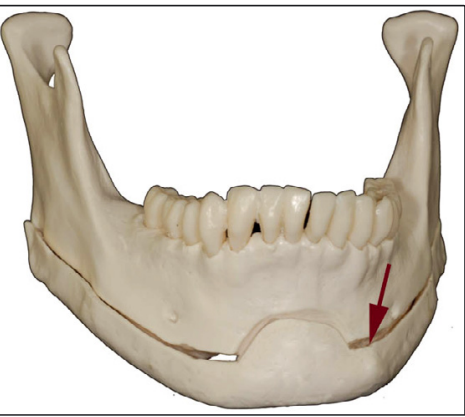

d

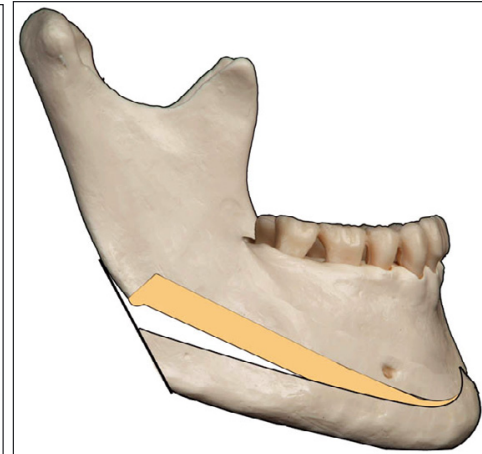

f

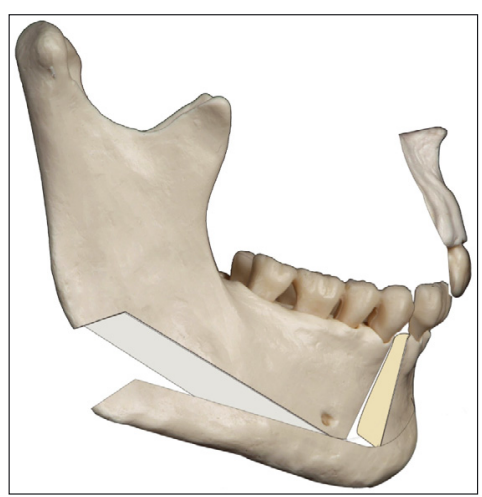

g

Figure 19

L'utilisation du chin wing permet d'intervenir sur le menton mais également sur l'ensemble du bord basilaire (a). Une fois le segment osseux découpé (b), sans effracter le périoste, celui-ci peut être déplacé dans le sens antéro-postérieur (c), pivoté dans le sens frontal pour corriger une asymétrie faciale (d), remonté pour corriger une hyperdivergence (e), abaissé pour corriger une hypodivergence $(\mathrm{f}, \mathrm{g})$. 


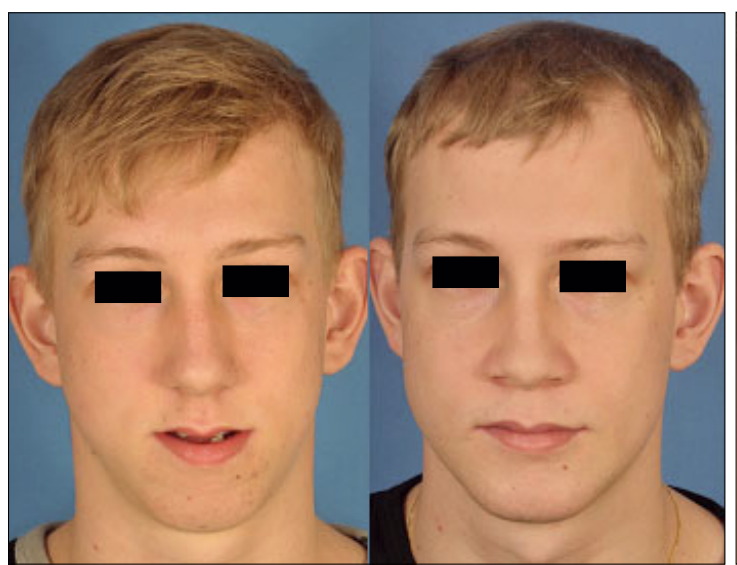

a

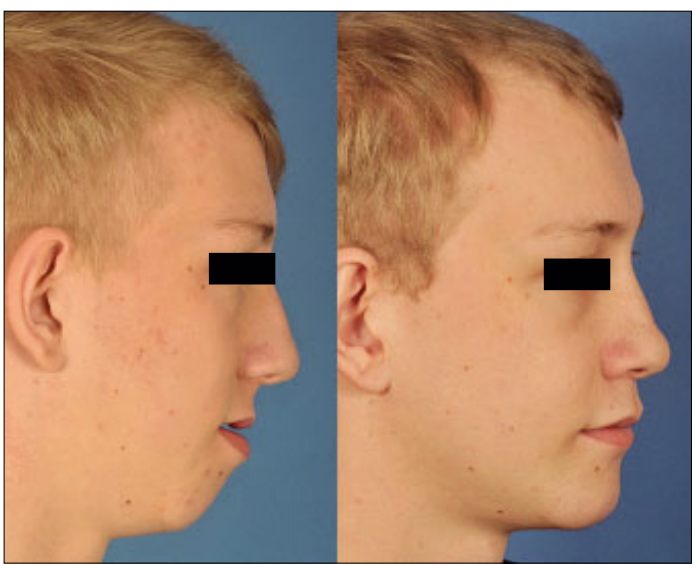

b

Figure 20

Une simple intervention de chin wing permet d'obtenir une compétence labiale au repos et un remodelage du bord mandibulaire. Cas $n^{\circ} 1$ : vues de face (a) et vues de profil (b) avant et après intervention du chin win.

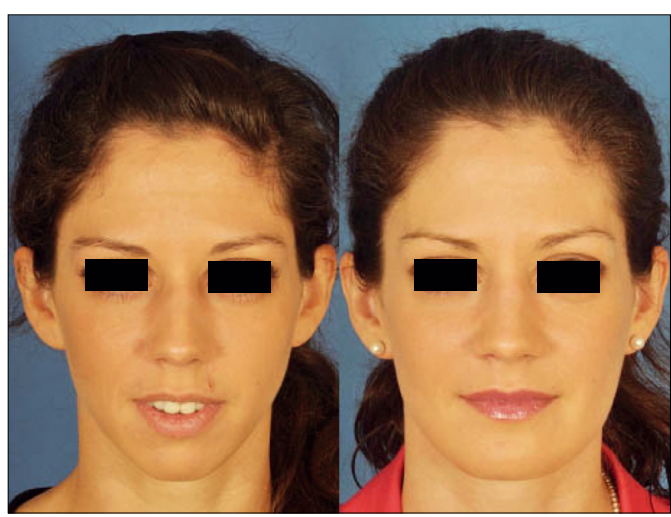

a

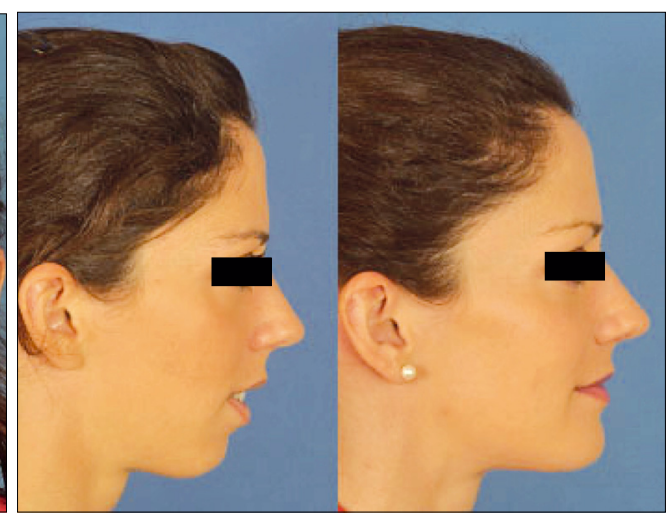

b

Figure 21

Cas $\mathrm{n}^{\circ} 2$ avec intervention de chin win. Vues de face (a) et vues de profil (b) avant et après chin win.

Courbe de Spee (Figs. 23a, 23b) La distraction du segment frontal en combinaison avec une ostéotomie de Le Fort I permet d'éviter une modification trop radicale du plan maxillaire. Lorsque la Courbe de Spee est trop marquée, le segment antérieur peut être mobilisé vers le bas (Fig. 23c-23g).

En cas d'hyperdivergence squelettique, une intervention de Le Fort I associée à une chin wing génioplastique pour modifier le bord basilaire est envisageable (Fig. 24). L'ostéotomie de chin wing peut permettre à elle seule et, dans certains cas, de rétablir une compétence labiale. Langle et le contour mandibulaires seront bien plus harmonieux qu'avec une ostéotomie sagittale (Figs. 20, 21).

S.R. : Qu'en est-il de la stabilité?

A.T. : Modifier le plan d'occlusion avec la distraction du segment frontal est parfaitement stable.
J'ai modifié des plans d'occlusion à l'aide de cette intervention sur plus de 1500 cas, et je n'ai aucun problème de stabilité.

Lintervention est peu invasive. Elle est réalisée sous anesthésie locale en $30 \mathrm{~min}$. Le distracteur dento-porté est mis en place une semaine après l'intervention. Une semaine après la fin de l'activation, le distracteur est déposé et remplacé par un arc lingual ou un arc transpalatin. Le traitement multiattache peut débuter immédiatement.

D’une façon générale, plusieurs facteurs importants sont à contrôler pour s'assurer de la stabilité :

1- Tout d'abord, comme je te l'ai dit, ne pas créer de compression condylienne.

2- Ensuite, ne jamais compenser orthodontiquement avant une intervention. Par exemple, ne pas chercher à fermer une béance 


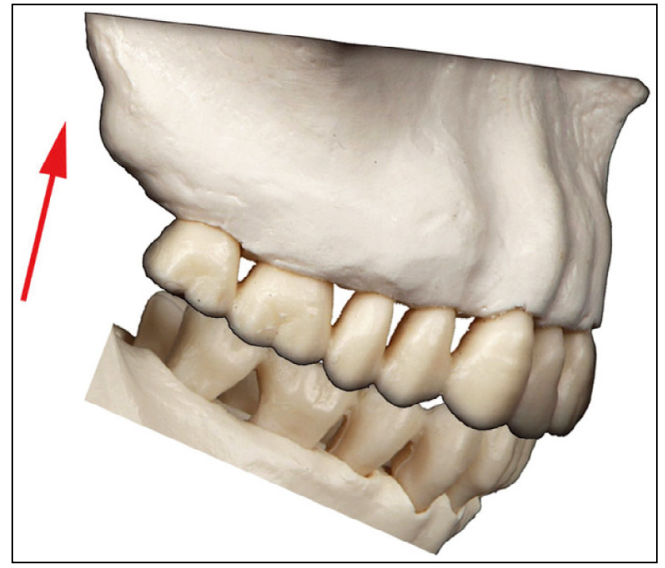

a

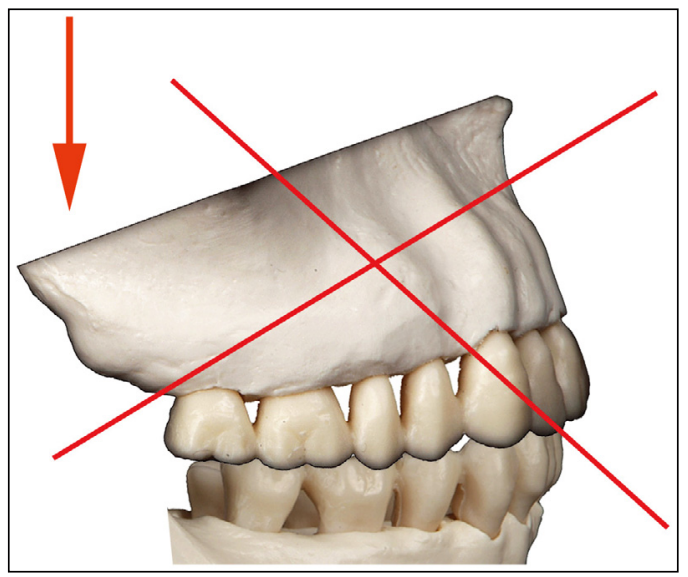

b

Figure 22

La bascule du plan occlusal peut se faire dans le sens des aiguilles d'une montre (a), mais pas dans le sens inverse (b).

orthodontiquement, mais plutôt accentuer encore plus l'infraclusion. Pour traiter ces infraclusions, il est possible de segmenter le maxillaire en trois pièces sans fixer le segment frontal, puis l'orthodontiste peut aisément fermer la béance en tractant l'ensemble du segment alvéolo-dentaire à l'aide de ses arcs orthodontiques en accentuant la supraclusion pour éviter les risques de récidive.

3- Ne pas compenser transversalement. Compenser orthodontiquement dans le sens transversal va créer, par la version vestibulaire des molaires, des risques de béance. Il est toujours aisé de placer un petit distracteur en même temps qu'une chirurgie de Le Fort I pour régler le problème transversal.

S.R. : Comment résous-tu le problème de compétence labiale lorsque le patient présente une lèvre supérieure courte?

A.T. : Il s'agit d'un problème effectivement difficile à résoudre car, lorsque tu impactes le maxillaire, tu remontes la lèvre supérieure en même temps. Donc, lorsque je réalise un Le Fort, j'effectue simultanément un lambeau, "le spina flap » sur lequel je vais publier prochainement.

S.R. : Lorthodontiste peut parfois hésiter à faire opérer des patientes en classe II squelettique, face courte, aux angles mandibulaires marqués, par crainte de masculiniser le profil. Quelles sont les options thérapeutiques pour des patientes présentant ce type facial?
A.T. : Tu peux sans souci envisager une ostéotomie sagittale avec une bascule dans le sens des aiguilles d'une montre pour effacer le menton et y associer une intervention de chin wing (Fig. 25).

S.R. : Lorsqu'un patient présente une classe III squelettique par prognathie mandibulaire, nous pouvons avoir des réticences à envisager un recul mandibulaire, au risque de diminuer la longueur sous-mentale, créer un «double menton » et aggraver les paramètres du sommeil. Quelles techniques envisages-tu pour le traitement de tel(le)s patient(e)s lorsqu'un recul mandibulaire est indiqué?

A.T. : Dans les très rares cas de prognathie mandibulaire franche, avec un surplomb inversé marqué, je vais opter pour un recul mandibulaire modéré et une avancée maxillaire par Le Fort I. Le recul seul devient de plus en plus exceptionnel car il peut générer un risque d'apnée du sommeil.

En fait, le patient se sent concerné par la longueur de sa mandibule [13]. Si tu modifies cette apparente longueur en la «cassant » grâce au chin wing, le patient a tout à coup l'impression d'avoir une mandibule plus petite (Fig. 26). Tu résous en même temps le problème du double menton. Je n'effectue quasiment plus de recul mandibulaire depuis que j'ai recours au chin wing que j'associe au Le Fort I.

Lorsque j'avance le maxillaire avec un Le Fort I, je prête une attention toute spéciale au seuil narinaire. Pour ne pas élargir la base du nez, j’ai imaginé cette intervention : après avoir incisé l'épine nasale antérieure, je remonte le trait d'incision le long du cartilage et je fixe la pointe du nez au niveau du cartilage 


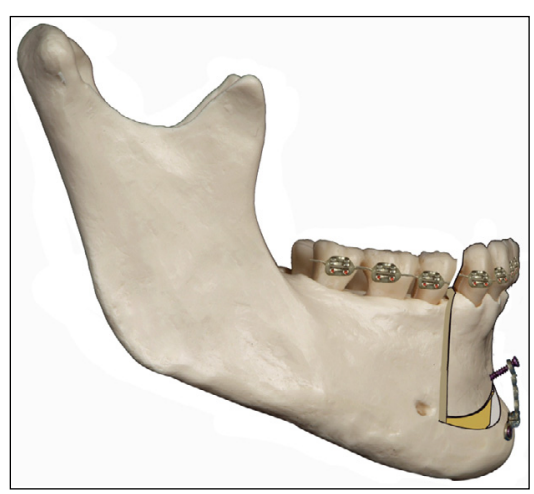

a

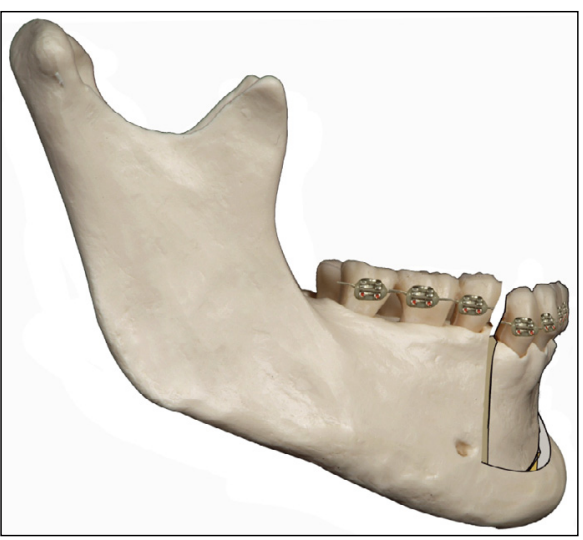

d

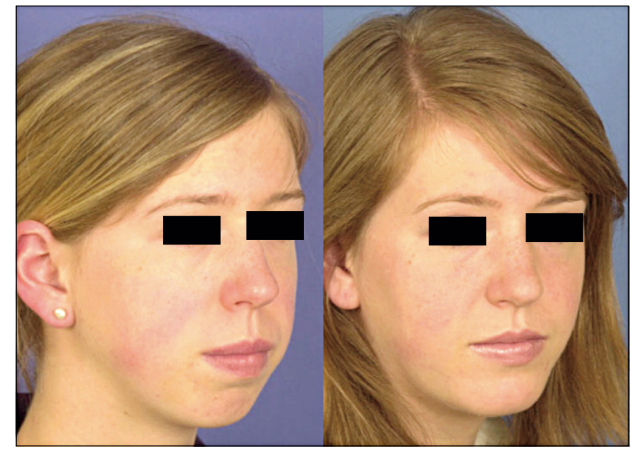

f

b
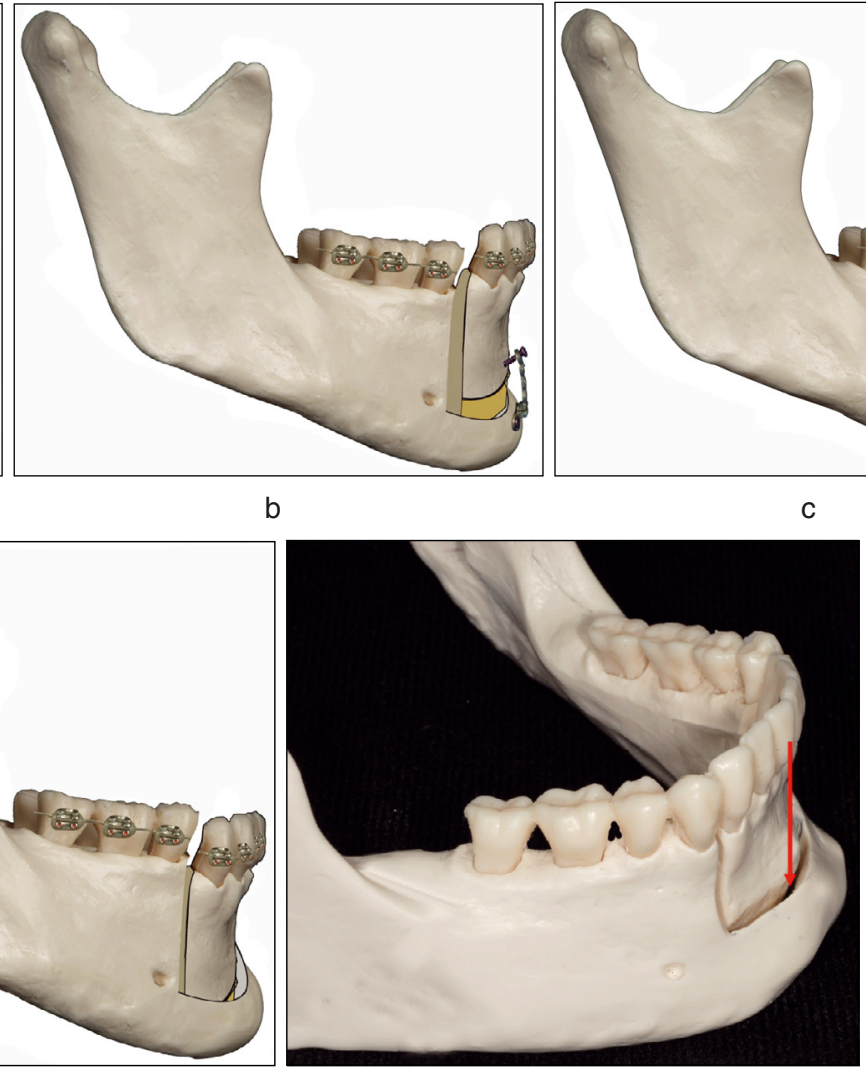

e

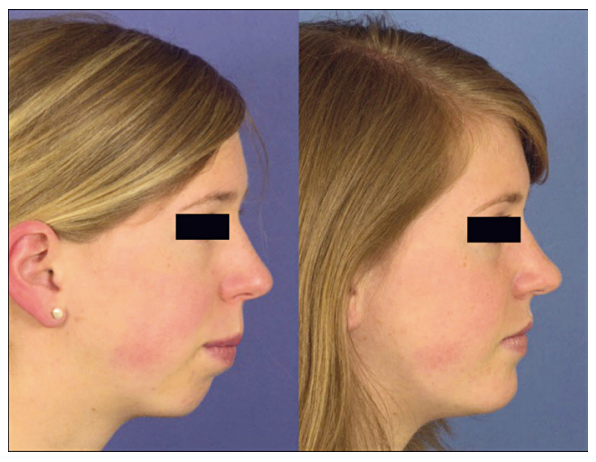

g

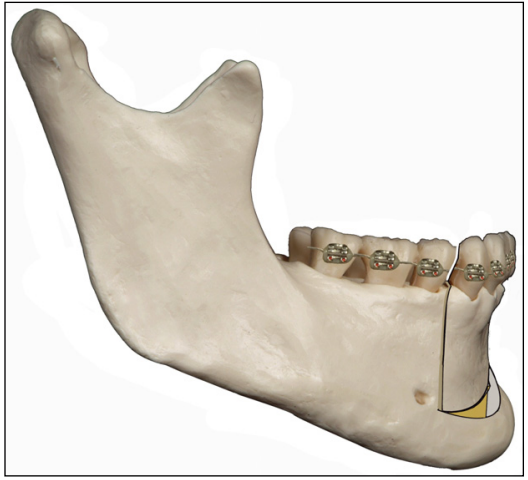

C

Figure 23

Infraclusion antérieure simple fermée par distraction partielle verticale antérieure du segment frontal (a-c). Lorsque la Courbe de Spee est trop marquée, le segment antérieur peut être mobilisé vers le bas $(d, e)$. Photographies extra-buccales avant et après distraction, de trois quart (f) et de profil $(g)$.

pour la remonter. Il est également important de gérer la liaison entre le nez et la lèvre supérieure sans créer de dépression au niveau de cette zone.

S.R. : Selon toi, comment traiter un patient présentant une forte asymétrie mandibulaire?

A.T. : Je contrôle toujours les condyles par scintigraphie. Si l'examen témoigne d'une différence d'activité entre les deux condyles, j'attends. En Suisse, les interventions ne sont remboursées qu'avant 20 ans. On est parfois obligés d'intervenir trop tôt pour cette raison. Je préviens alors les parents et le patient que je serai probablement dans l'obligation de réintervenir plus tard.

\section{S.R. : Et plus tôt ?}

A.T. : Non, jamais. J'estime que l'ostéotomie du condyle est invasive. Un fois la croissance achevée, il 


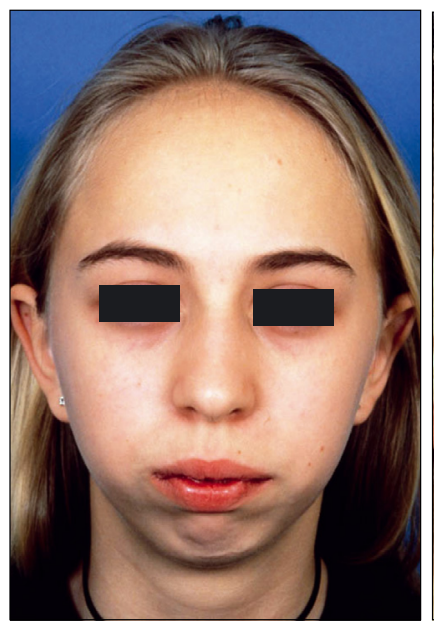

a

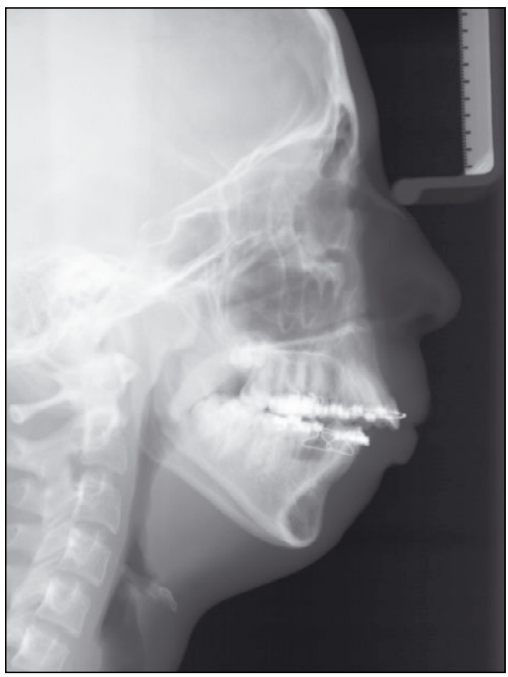

C

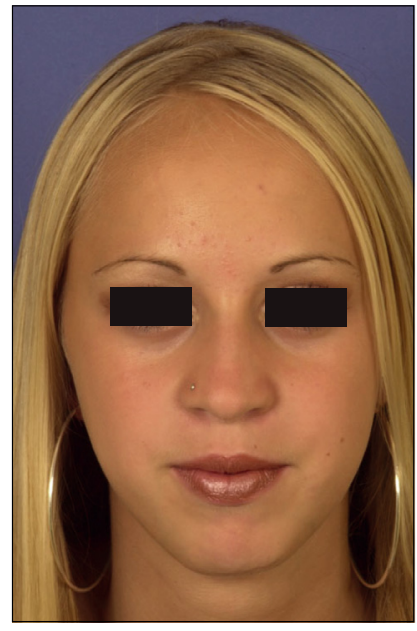

e

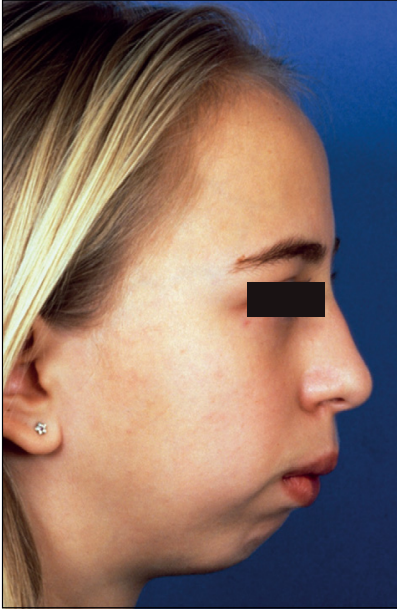

b

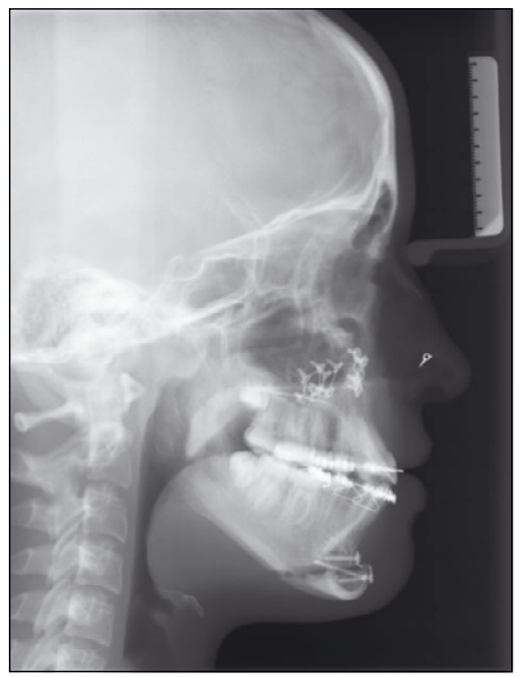

d

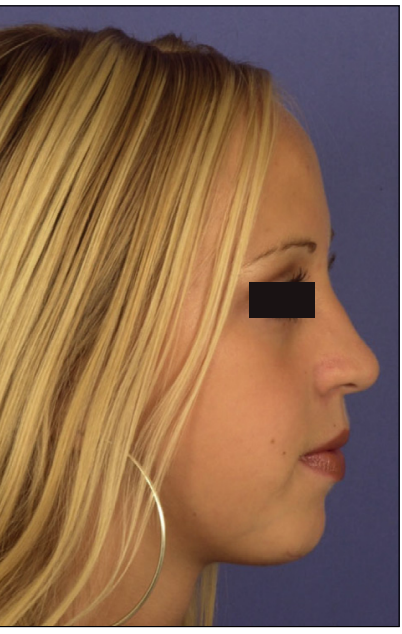

f

Figure 24

Patiente traitée sans extraction. La biproalvéolie donnant un aspect disgracieux du fait de l'hyperdivergence et de la rétrogénie n'est pas corrigée. Une intervention de Le Fort avec impaction associée à une génioplastie équilibre le visage sans avoir recours aux extractions. Vues de face (a) et de profil (b) en début de traitement. Radiographies en début (c) et fin (d) de traitement. Vues de face (e) et de profil (f) en fin de traitement. 


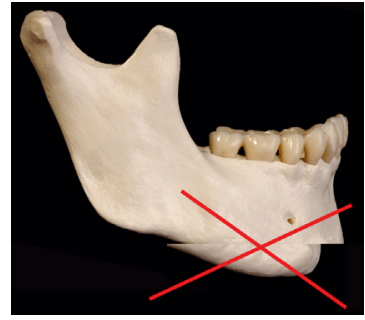

a

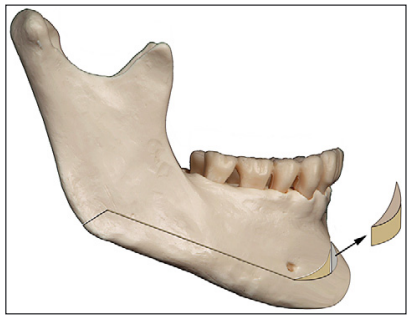

e

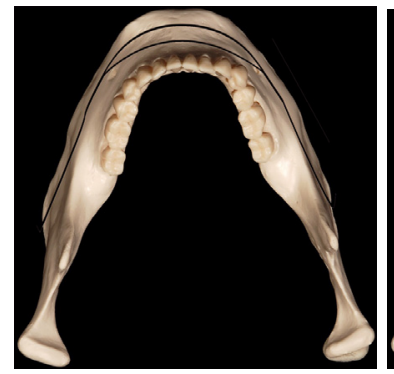

i

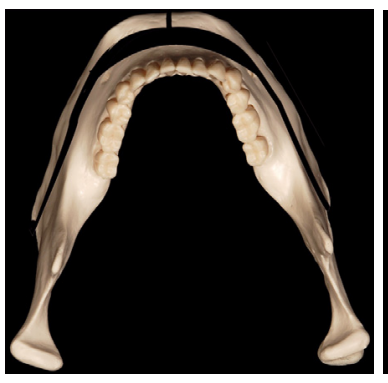

$\mathrm{m}$

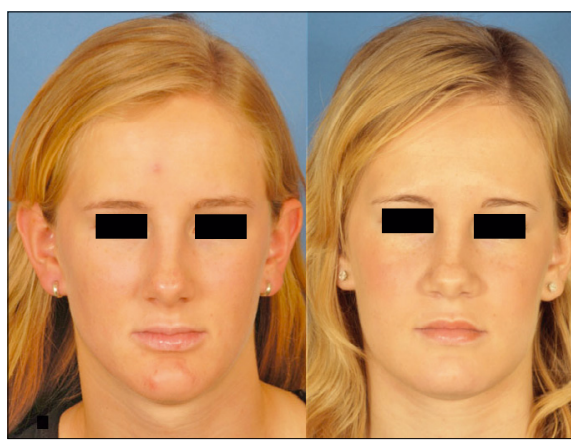

$p$

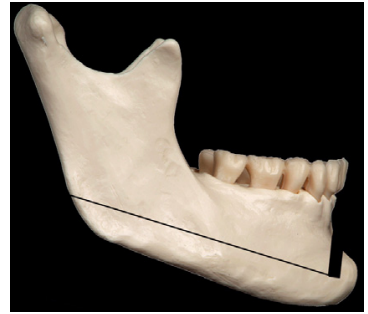

C
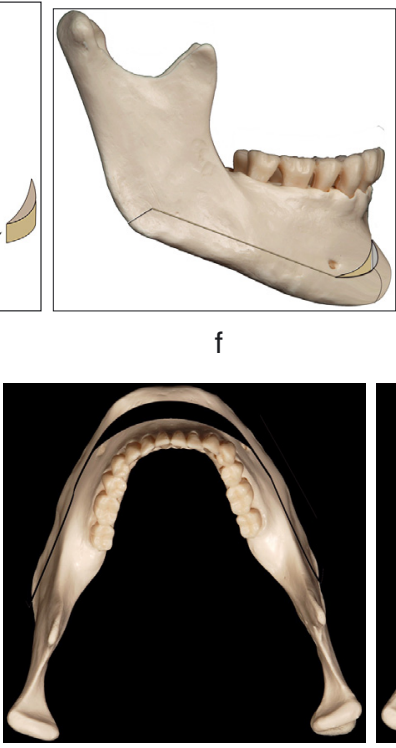

j

g

k

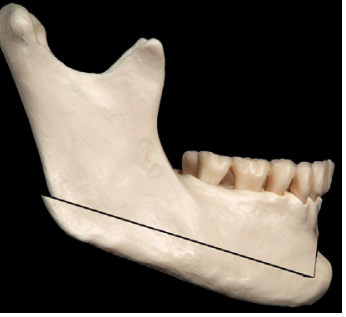

d
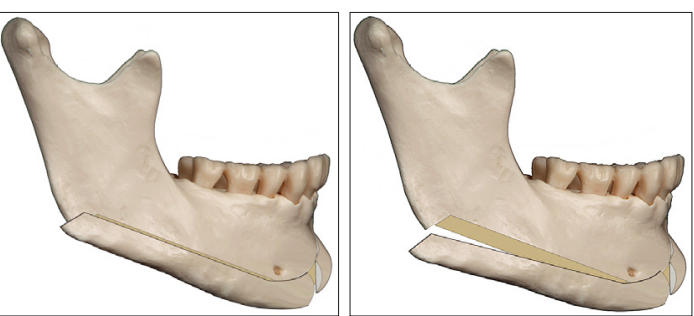

h
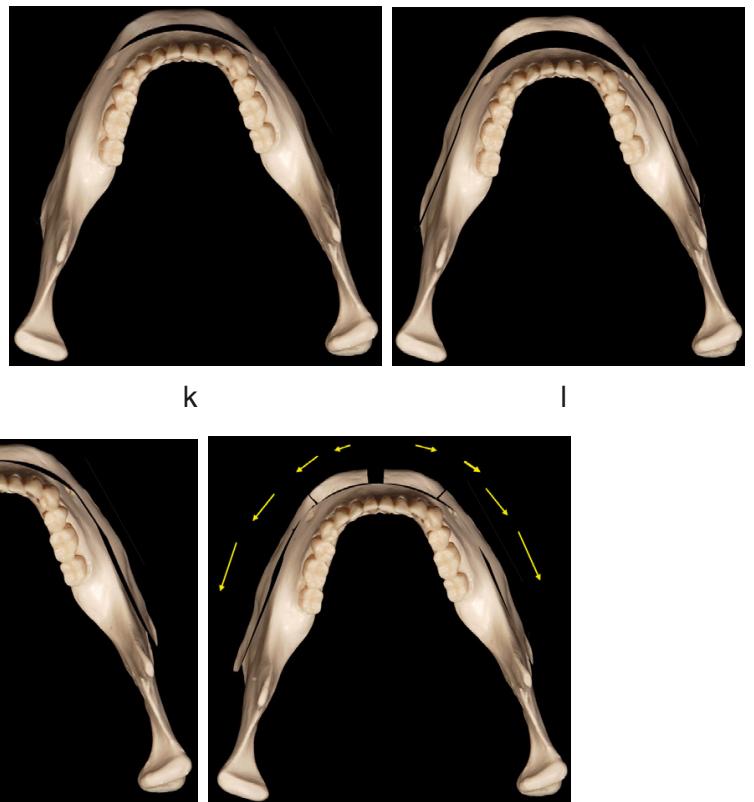

o

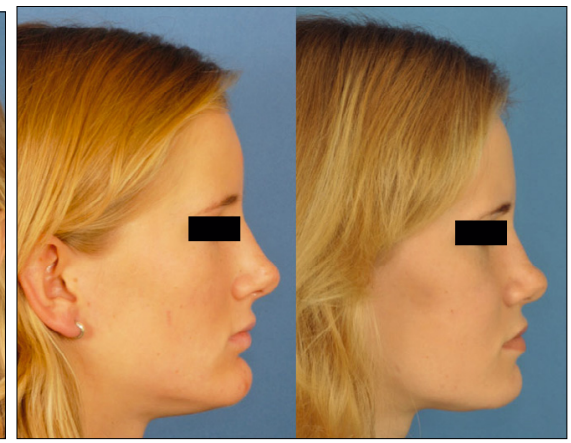

q

Figure 25

L'intervention de chin wing offre la possibilité de modifier la forme du menton dans les trois dimensions de l'espace : antéropostérieure, verticale et transversale. Elle permet notamment d'effacer et d'arrondir le menton. Vues sur moulages des différentes étapes de l'ostéotomie (a à o). Associée à une avancée mandibulaire, une patiente présentant une classe II squelettique hypodivergente présente, après traitement, un profil doux et féminin $(p, q)$. 


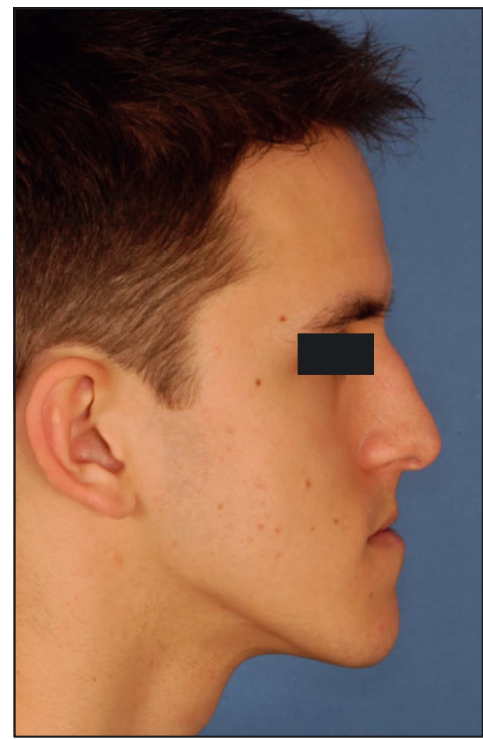

a

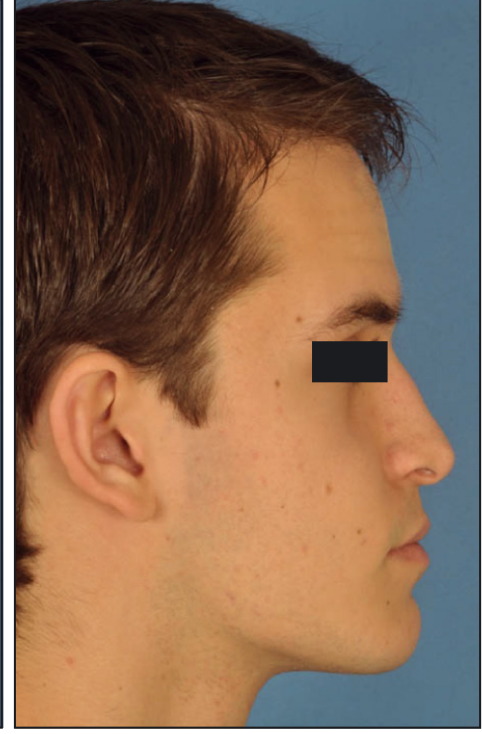

b

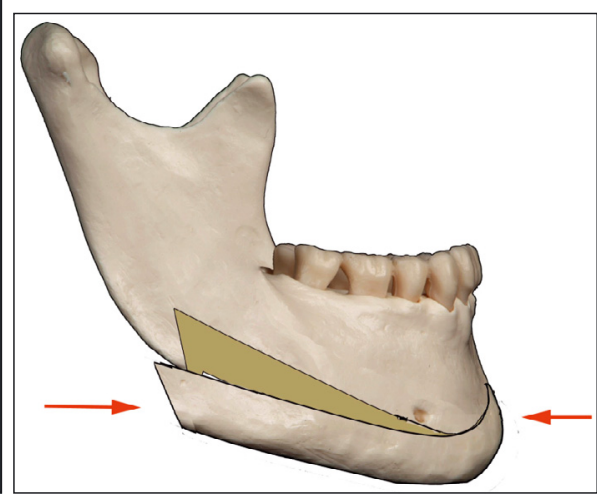

C

Figure 26

L'intervention de chin wing en « cassant » la longueur de la mandibule (a et c) permet de réduire l'hyperdivergence squelettique et de corriger une classe III squelettique uniquement par avancée maxillaire (b).

est aisé de réaliser une ostéotomie sagittale [8], éventuellement associée à un chin wing.

S.R. : Tu as insisté sur l'apport de la chirurgie maxillo-faciale pour résoudre les problèmes d'apnée du sommeil. Tu as notamment beaucoup réfléchi sur l'amélioration de la ventilation nasale. Le recours à des interventions classiques (septoplastie, turbinoplastie) ne présente pas, selon toi, que des avantages car elles provoqueraient des épaississements de la muqueuse nasale gênant la ventilation surtout dans le secteur paraseptal.

Tu proposes une intervention au niveau du seuil narinaire [11]. Peux-tu nous parler des indications et des avantages de cette technique? Tu n'as pas eu le temps de nous décrire le recours à la mise en place de «ballonnets». Peux-tu nous en dire plus sur ce sujet?

A.T. : La chirurgie maxillo-faciale aide à la résolution des problèmes d'apnée du sommeil, parce que le chirurgien maxillo-facial en avançant les maxillaires (Fig. 27), ou en les augmentant transversalement a un impact direct sur la perméabilité des voies aériennes supérieures. L'expansion transversale progressive du maxillaire (SARPE) génère (par l'écartement des parois latérales des fosses nasales, donc de leur volume), chez la plupart des patients une amélioration de la ventilation nasale. Concernant septoplastie et turbinoplastie, j'aimerais préciser qu'intervenir sur le septum n'altère pas le développement facial. J'ai vu Millard opérer des nez d'enfants de 6-7 ans. En revanche, je n'interviens au niveau du septum que s'il est réellement obstructif, sinon je préfère envisager une latéralisation de la paroi externe de l'orifice piriforme. Lorsque tu redresses le septum, il est toujours épaissi après. Il faut savoir que lorsque tu réalises une intervention ORL conventionnelle, $10 \%$ des patients inhalent moins bien qu'avant car les interventions altèrent la muqueuse nasale. Or, la muqueuse nasale est très délicate. Mon intervention de latéralisation des parois nasales se réalise sans décollement de la muqueuse nasale.

S.R. : En quoi consiste cette intervention de latéralisation de la paroi de l'orifice piriforme?

A.T. : Tu débutes l'intervention par une incision au niveau du vestibule en regard de l'aile du nez, tu atteins l'orifice piriforme dont tu découpes la paroi externe au niveau de la base de l'orifice. Ensuite, tu peux poursuivre et balayer ton incision de haut en bas sans affecter la muqueuse et tu déplaces délicatement le cornet vers le sinus. Beaucoup d'ORL m'envoient leurs patients pour ce type d'intervention car les patients ventilent vraiment mieux après (Fig. 28).

S.R. : Qu'est-ce que cet appareil avec une pompe et un «ballonnet » sur ton bureau?

A.T. : Oui, regarde, c'est Noël, je viens de le recevoir! C'est mon nouvel instrument pour augmenter la perméabilité nasale! Il s'agit d'un ballonnet de 


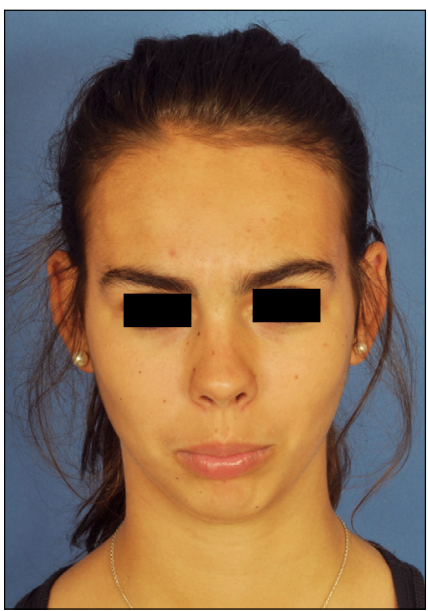

a

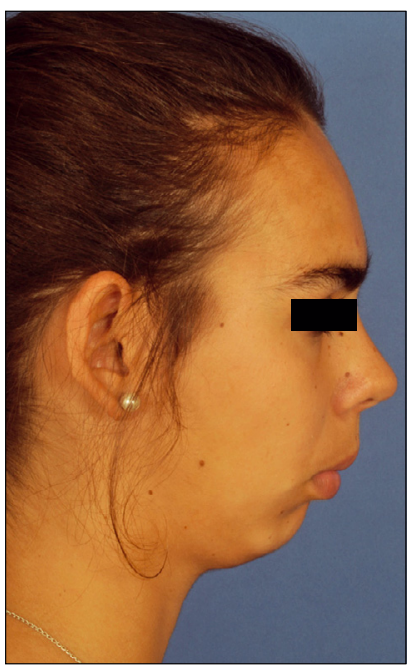

C

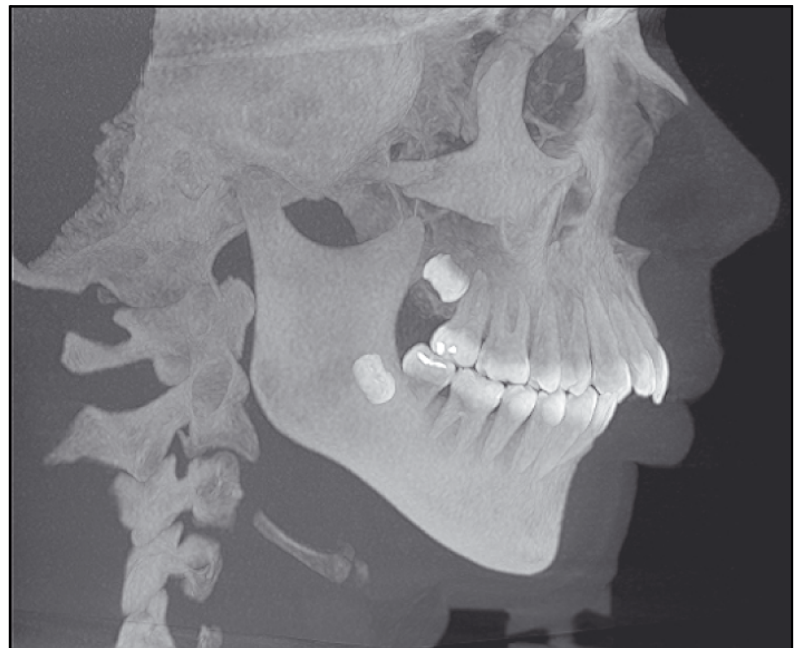

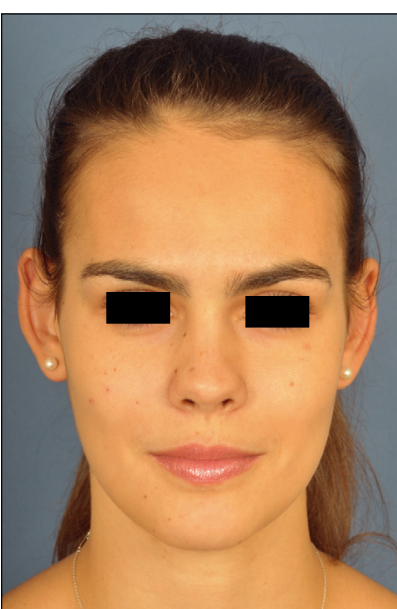

b

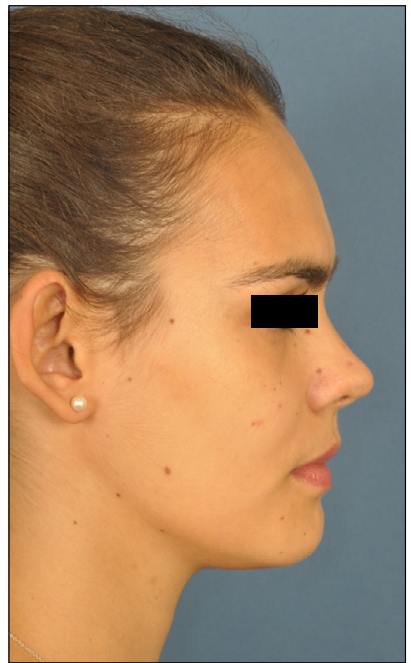

d

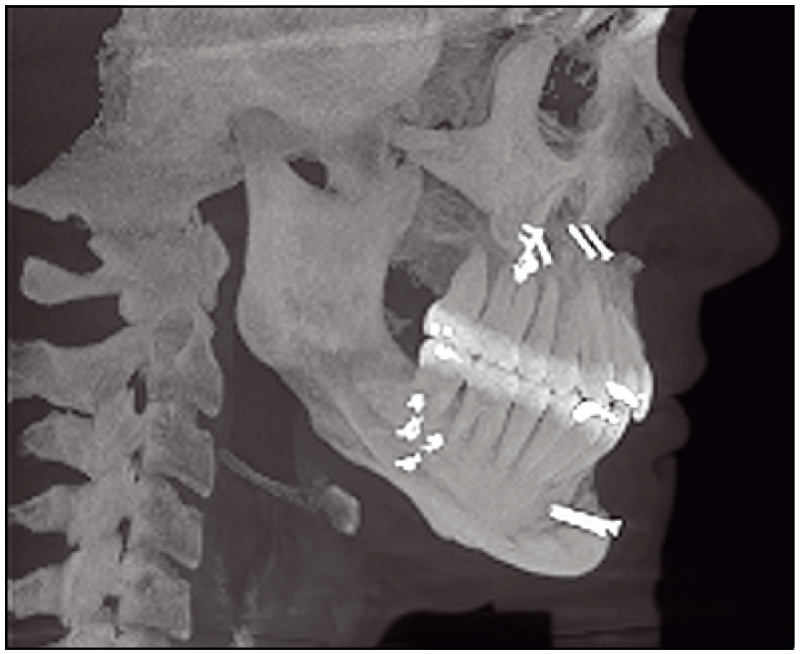

Figure 27

Résolution des problèmes d'apnée du sommeil par l'augmentation de volume : Le Fort I d'avancée, ostéotomie sagittale et chin wing. Vues de face avant (a) et après (b) traitement. Vues de profil avant (c) et après (d) traitement. Radiographies de profil avant (e) et après ( $f$ ) traitement. 


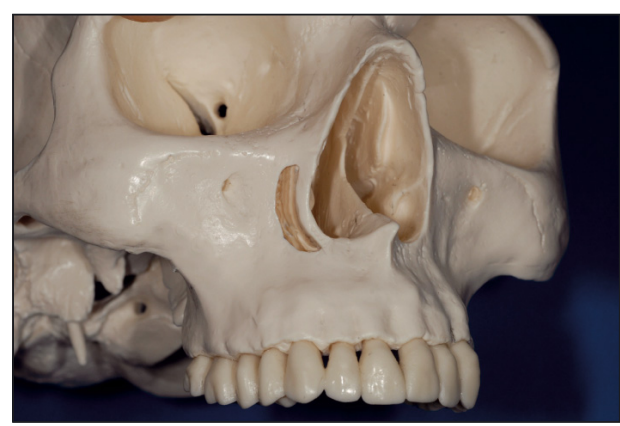

a

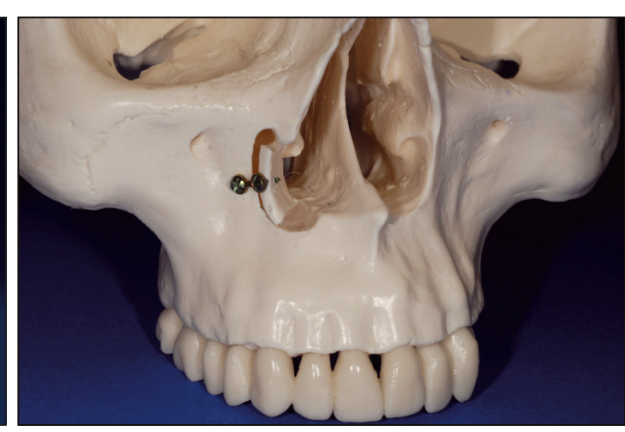

b

Figure 28

( $a$ et b) Latéralisation de la paroi externe de l'orifice piriforme.

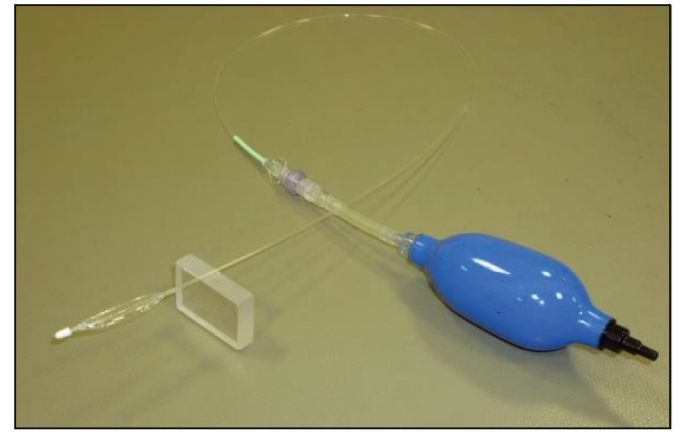

a

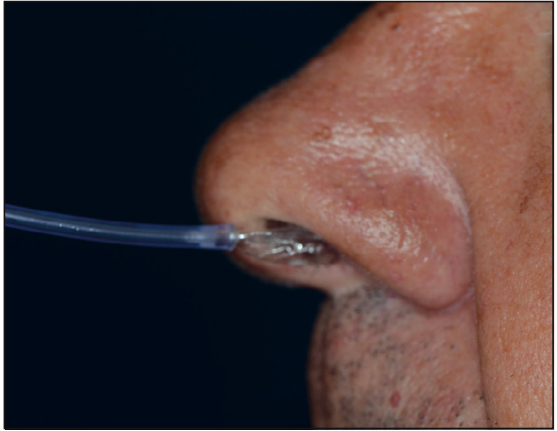

b

Figure 29

Ballonnets, vue de l'appareil (a) et mise en situation (b).

$3 \mathrm{~cm}$ de long réalisé dans un matériau très doux et très fin de façon à ne créer aucune sensation de gêne lorsque tu l'insères et que tu le gonfles, à l'aide de la petite pompe, dans la narine. Tu peux enduire les ballonnets de la crème prescrite par l'ORL et la pression du ballonnet sur la muqueuse va favoriser la pénétration de la crème (Fig. 29).

S.R. : Tu les gardes combien de temps?

A.T. : Trois minutes par jour pendant trois mois.

S.R. : Ça marche? Quand les préconises-tu?

A.T. : Pour l'instant, je ne les ai essayés que sur moi. Ca a énormément amélioré ma ventilation. Je les préconiserais dans un premier temps, pour tenter d'éviter une intervention chirurgicale.

S.R. : Une des grandes questions sociétales actuelles est celle du vieillissement. Quelle est ta vision d'un visage qui «vieillit bien»?

A.T. : Difficile de répondre à cette question, car hélas il existe une ptose des tissus avec l'âge que l'on peut difficilement contrôler sauf par le lifting qui est malgré tout un acte assez invasif. La combinaison d'une chirurgie d'augmentation de volume, type wing et d'un lifting peut donner des résultats parti- culièrement jolis. À notre échelle, il faut positionner les dents de façon à ce que les incisives maxillaires soient le plus longtemps visibles lors du sourire, de façon à prévenir l'effacement du vermillon de la lèvre.

S.R. : Dans la clinique Pyramide am See de Zurich où tu exerces, comment organises-tu le parcours du patient entre le cabinet de ton correspondant orthodontiste et toi-même?

A.T. : Tu sais, je travaille avec beaucoup d'orthodontistes, pas uniquement celui de la clinique. J'aime bien recevoir le patient en premier et avoir une idée précise de ce que j'aimerais demander à l'orthodontiste. En tous les cas, les échanges doivent débuter avant que l'on initie le traitement. Lorthodontiste a une notion bien précise de ses objectifs et des limites liées aux particularités du cas. Il en va de même pour le chirurgien. Quand l'orthodontiste n'exerce pas loin, il (elle) se déplace à mon cabinet et l'on discute de vive-voix des détails. Lorsque ce n'est pas le cas, on utilise le programme Team Viewer 10 qui nous permet en direct de partager les éléments de 
nos dossiers respectifs et de planifier le traitement ensemble.

Lorsqu'il s'agit d'orthodontistes d'autres pays, qu'ils m'adressent des cas déjà préparés, ce n'est pas idéal, mais à présent, avec tous les types d'intervention à notre disposition, on parvient toujours à résoudre le problème esthétique, occlusal et fonctionnel.

S.R. : Dans nos professions, nous pouvons être sujets aux dogmes, aux "tout pour", "tout contre", aux retours de balancier. Que signifie pour toi la recherche de l'équilibre?

A.T. : Lorsque j'ai commencé à publier, je remettais en question certains dogmes et autres certitudes et proposais mes propres solutions. Mes confrères m'ont fait comprendre que j'étais un hurluberlu. J'étais le mouton noir. Petit à petit, avec le recul, certains confrères ont adopté quelques unes de mes techniques. Je suis devenu un mouton gris. Aujourd'hui, beaucoup de mes confrères pratiquent les interventions que j'ai développées. Nous sommes tous des moutons blancs à présent! (Fig. 30).

Tu vois par exemple, le recours à l'implant palatin comme ancrage, je l'ai publié en février 1992 avec Michele Antonini [1]. Aujourd'hui combien d'orthodontistes l'utilisent!

\section{S.R. : Quant à la recherche de l'équilibre?}

A.T. : C'est difficile de répondre de but en blanc à cette question car chaque personne a un point de vue différent. Pour moi, c'est réussir à allier le fonctionnel à l'esthétique, c'est rechercher des solutions efficaces pour résoudre les problèmes spécifiques à chaque patient et sans cesse étudier, réfléchir, se renouveler.

S.R. : Peux-tu nous faire partager ta vision de l'évolution future de la spécialité de CMF?

A.T. : Mon violon d'Ingres aujourd'hui est la suppression des cicatrices. Jusqu'à présent, lorsque l'on intervenait pour supprimer les cicatrices, on en créait d'encore plus grandes. J'ai reçu une patiente de Singapour qui avait bénéficié de plasties de sa cicatrice. La cicatrice était bien plus longue qu'au départ. Je lui ai proposé de provoquer une régénération de son épiderme par distraction.

J'ai également utilisé ce procédé sur une patiente opérée jeune mais présentant une fente résiduelle au niveau du palais. Avec ce nouvel appareil que j'ai créé, l'espace résiduel a été recouvert d'épithélium

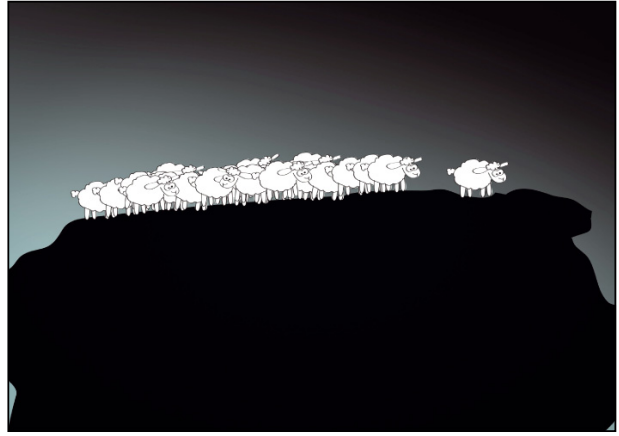

Figure 30

Albino Triaca, du fait de ses inventions, paraissait à l'époque être un original, un mouton noir. Ses théories à présent reconnues par ses pairs, il est considéré comme un mouton blanc.

en deux semaines. Cette technique sera publiée en détail prochainement.

S.R. : Mais tu m'as parlé d'un autre type d'intervention au niveau des cicatrices chéloïdes des fentes palatines. . .

A.T. : Oui, à l'aide d'un tube extrêmement fin dont l'extrémité est affutée, je passe au sein de la cicatrice. Puis je prélève un greffon au niveau du tissu conjonctif de la muqueuse palatine et j'insère le tissu conjonctif délicatement dans le tunnel créé sous la cicatrice.

S.R. : Cela ressemble aux techniques de tunnelisation utilisées en parodontologie?

A.T. : Oui, tout à fait. Les résultats au niveau des cicatrices sont spectaculaires.

S.R. : Et l'utilisation de cellules souches?

A.T. : Oui, les cellules souches, les transplantations... Ce sont des voies d'avenir pour prévenir et solutionner de nombreux problèmes de régénération tissulaire.

S.R. : Le philosophe Levinas* écrivait que dès que nous observons le visage d'un individu et sommes capable de le décrire, nous le dépersonnalisons; l'individu devient objet « La meilleure manière de rencontrer autrui, c'est de ne pas même remarquer la couleur de ses yeux!" Toi qui analyses les éléments de la face quasi instantanément, qui est capable d'assembler différents segments d'os comme le fait un ébéniste pour modeler les visages à ta guise, penses-tu, comme Levinas, que cette aptitude professionnelle peut modifier la relation sociale que tu entretiens avec tes patients, avec ton entourage ? ${ }^{2}$

${ }^{2}$ Emmanuel Levinas. Totalité et Infini. Ed : Livre de Poche, 1971. 
A.T. : C'est possible... Avec l'expérience, on prend du recul...

Nos patients ont une demande que je tente de satisfaire. Comme je suis un passionné, je recherche toujours des solutions nouvelles pour résoudre demain des détails qui ne me satisfont pas aujourd'hui.

S.R. : Merci Albino pour ton extraordinaire et permanente contribution à l'amélioration du bien-être physique et fonctionnel de nos patients $[9,14,15]$.

\section{Remerciements}

Remerciements à Christian Paulus pour la traduction de certaines réponses rédigées en allemand.

\section{Conflit d'intérêt}

Les auteurs déclarent n'avoir aucun lien d'intérêt concernant les données publiées dans cet article.

\section{Bibliographie}

[1] Grimm G. Die intraorale Operation der asymmetrischen Kinnregion. Fortschr Kiefer Gesichtchirur 1961;7:173.

[2] Guerrero CA, Bell WH, Contasti G, Rodriguez AM. Mandibular widening by intraoral distraction osteogenesis. Br J Oral Maxillofac Surg 1997;35(6):383-392.

[3] Gunbay T, Akay MC, Aras A, Gomel M. Effects of transmandibular symphyseal distraction on teeth, bone and temporomandibular joint. J Oral Maxillofac Surg 2009;67:2254-2265.

[4] Joss CU, Triaca A, Antonini M, Kiliaridis S, KuijpersJagtman AM. Skeletal and dental stability of segmental distraction of the anterior mandibular alveolar process. A 5.5-year follow-up. Int J Oral Maxillofac Surg 2013;42:337-344
[5] Joss CU, Triaca A, Antonini M, Kiliaridis S, KuijpersJagtman AM. Soft tissue stability after segmental distraction of the anterior mandibular alveolar process: a 5.5 year follow-up. Int J Oral Maxillofac Surg 2013;42: 345-351.

[6] Merli M, Merli M, Triaca A, Esposito M. Segmental distraction osteogenesis of the anterior mandible for improving facial esthetics. Preliminary results. World J Orthod 2007;8:19-29.

[7] Moorees CF, Gron AM. Principles of orthodontic diagnosis. Angle Orthod 1966;6:25-62.

[8] Triaca A. Dentobasal correction of mandible with a bilateral bisagittal split. Inf Orthod Kieferorthop 1989;21:425-432.

[9] Triaca A, Antonini M, Minoretti R, Merz BR. Segmental distraction osteogenesis of the anterior alveolar process. J Oral Maxillofac Surg 2001;59:26-34; discussion 34-35.

[10] Triaca A, Antonini M, Wintermantel E. Ein neues Titan-Flachschrauben-Implantat zur ortodontischen Verankerung am anterioren Gaumen. Inf Orthod Kieferorthop 1992;24:251-257.

[11] Triaca A, Brusco D, Guijarro-Martínez R. Nasal wall lateralization: a novel technique to improve nasal airway obstruction. Br J Oral Maxillofac Surg 2013;51: e24-e25.

[12] Triaca A, Brusco D, Guijarro-Martínez R. Chin wing osteotomy for the correction of hyperdivergent skeletal class III deformity: technical modification. Br J Oral Maxillofac Surg 2015;53:775-777.

[13] Triaca A, Garibaldi J, Simonetti M, Balbi P, Laffi N, Merlini A. Reconstruction and rehabilitation of severe mandibular atrophy using distraction osteogenesis technique: a case report. J Oral Maxillofac Surg 2014;72(12):2405-2418.

[14] Triaca A, Minoretti R, Merz B. Treatment of mandibular retrusion by distraction osteogenesis: a new technique. $\mathrm{Br}$ J Oral Maxillofac Surg 2004;42(2):89-95.

[15] Triaca A, Minoretti R, Saulacic N. Mandibular wing osteotomy for correction of the mandibular plane: A case report. Br J Oral Maxillofac Surg 2010;48:182-184.

[16] Triaca A, Furrer T, Minoretti R. Chin shield osteotomy a new genioplasty technique avoiding a deep mento-labial fold in order to increase the labial competence. Int J Oral Maxillofac Surg 2009;38:1201-1205. 